\title{
Inhibition of Rho Kinase Induces Antioxidative Molecules and Suppresses Reactive Oxidative Species in Trabecular Meshwork Cells
}

\author{
Tomokazu Fujimoto, ${ }^{1}$ Toshihiro Inoue, ${ }^{1}$ Saori Ohira, ${ }^{1}$ Nanako Awai-Kasaoka, ${ }^{1}$ \\ Takanori Kameda, ${ }^{2}$ Miyuki Inoue-Mochita, ${ }^{1}$ and Hidenobu Tanihara ${ }^{1}$ \\ ${ }^{1}$ Department of Ophthalmology, Faculty of Life Sciences, Kumamoto University, 1-1-1 Honjo, Kumamoto 860-8556, Japan \\ ${ }^{2}$ Department of Ophthalmology and Visual Sciences, Kyoto University Graduate School of Medicine, Yoshida-Konoe-cho, Sakyo-ku, \\ Kyoto 606-8501, Japan \\ Correspondence should be addressed to Toshihiro Inoue; noel@da2.so-net.ne.jp
}

Received 21 February 2017; Revised 22 May 2017; Accepted 30 May 2017; Published 19 July 2017

Academic Editor: Ciro Costagliola

Copyright (c) 2017 Tomokazu Fujimoto et al. This is an open access article distributed under the Creative Commons Attribution License, which permits unrestricted use, distribution, and reproduction in any medium, provided the original work is properly cited.

\begin{abstract}
Purpose. To investigate the effect of rho kinase inhibitors on oxidative stress in trabecular meshwork (TM) cells. Methods. TM cells were isolated from the eyes of cynomolgus monkeys. Y-27632 and menadione were used to inhibit rho kinase and induce production of reactive oxygen species (ROS), respectively. The cynomolgus monkey array and 12,613 probes were used in DNA microarray analysis, and the affected genes were categorized using gene ontology analysis. The mRNA levels of the target genes were confirmed by real-time RT-PCR. Intracellular oxidative stress was detected using a fluorescent reagent sensitive to ROS. Cell viability was assessed by the WST- 8 assay. Results. Gene ontology analysis revealed upregulation of genes involved in antioxidant activity, and upregulation of catalase was confirmed by real-time RT-PCR after 30 min treatment with Y-27632. Production of ROS was increased by menadione, and the effect was partly suppressed by pretreatment with Y-27632. At a lower dose of menadione, Y-27632 stimulated TM cells and significantly increased their viability following menadione treatment compared to control cells. Conclusion. Using microarray analysis, Y-27632 was shown to upregulate antioxidative genes including catalase and partially reduce ROS production and cell death by oxidative stress caused by menadione.
\end{abstract}

\section{Introduction}

Oxidative stress is a major physiological phenomenon, mediated through the production of reactive oxygen species (ROS), such as peroxides, superoxide, hydroxyl radical, and singlet oxygen. ROS play an important role in cell homeostasis and pathogen response and are therefore essential in biological processes. In contrast, increases in ROS are seen in various age-related diseases including glaucoma [1]. For instance, in the aqueous humor of glaucoma patients, the levels of oxidative stress markers are significantly increased [2-5]. Additionally, oxidative DNA damage is reportedly increased in the trabecular meshwork (TM) of glaucoma patients $[6,7]$. These findings indicate that the TM of glaucomatous eyes is continuously exposed to oxidative stress, and therefore, damage to TM may increase outflow resistance and the risk of glaucoma progression. In line with this, lower systemic antioxidant capacity is related to higher intraocular pressure (IOP) levels in open-angle glaucoma patients [8]. Moreover, glaucoma-related genes, such as CYP1B1 and FOXC1, are reportedly linked to oxidative stress in the eyes [9-12]. Taken together, control of oxidative stress in the eye may be a therapeutic target to slow glaucoma progression.

Rho-rho kinase (ROCK) signaling controls polymerization of actin and thereby mediates various cell functions, such as contraction, migration, phagocytosis, and mitosis. Inhibition of ROCK increases aqueous outflow by depolymerizing F-actin in TM cells and Schlemm's canal endothelial cells $[13,14]$. A ROCK inhibitor, ripasudil, has been 
approved as an IOP-lowering drug in Japan [15]. Ripasudil significantly reduces the IOP of glaucoma patients upon either single or multiple administration $[16,17]$. However, ROCK inhibitors have drawn attention as antioxidative drugs against cardiovascular diseases and chronic renal injury $[18,19]$. Indeed, ripasudil (also known as K-115) has been reported to have a neuroprotective effect on the optic nerve by suppressing oxidative stress in an animal model [20]. Thus, the effect of ROCK inhibitors on oxidative stress in TM cells is of interest from a therapeutic point of view against glaucoma.

Here, we show the results of an exhaustive investigation using a microarray, revealing that treatment with Y-27632, a well-known ROCK inhibitor, upregulates antioxidative molecules in TM cells, inhibits ROS production, and promotes cell survival.

\section{Materials and Methods}

2.1. Cell Culture. Trabecular meshwork (TM) cells were isolated from the eyes of cynomolgus monkeys (Shin Nippon Biomedical Laboratories, Kagoshima, Japan) according to the method described previously [21]. Primary TM cells were cultured in Dulbecco's modified Eagle's medium (DMEM; Wako, Osaka, Japan) supplemented with 10\% FBS, $2 \mathrm{mM}$ glutamine, $100 \mathrm{U} / \mathrm{mL}$ penicillin, $100 \mu \mathrm{g} / \mathrm{mL}$ streptomycin, and $0.5 \mu \mathrm{g} / \mathrm{mL}$ amphotericin $\mathrm{B}$ at $37^{\circ} \mathrm{C}$ in $5 \% \mathrm{CO}_{2}$. These cells were used after 2-5 passages. The character of the isolated cells in the present study was confirmed by expression of specific TM markers (caveolin 1, collagen $4 \alpha 5$, matrix gla protein, tissue inhibitor of metalloproteinase 3 , and vascular cell adhesion protein 1), phagocytosis function, and myocilin induction by dexamethasone as described previously [22].

2.2. DNA Microarray Analysis. Custom cDNA microarray analysis was performed using a CombiMatrix microarray (CombiMatrix, Mukilteo, WA) as described previously [23]. Briefly, the cynomolgus monkey array was designed to detect directly labeled mRNA from 12,613 probes. Confluent TM cells in $100 \mathrm{~mm}$ dishes were treated with $25 \mu \mathrm{M} \mathrm{Y}-27632$ (Merck Millipore, Darmstadt, Germany) or vehicle (deionized water) for $30 \mathrm{~min}$. Total RNA was extracted from the cells, and the integrity and concentration of total RNA was measured using an Agilent 2100 Bioanalyzer (Agilent Technologies, Santa Clara, CA). Fluorescence-labeled antisense RNA was synthesized by direct incorporation of Cy5-UTP or Cy3-UTP, using each RNA sample and an RNA Transcript SureLABEL Core kit (Takara Bio, Shiga, Japan). Labeled antisense RNAs were hybridized simultaneously with the microarray chips. DNA microarray preparation, hybridization, processing, scanning, and analyses were performed according to the manufacturer's instructions (Filgen, Nagoya, Japan). Fluorescent images of hybridized microarrays were obtained with a GenePix 4000B Scanner (Molecular Devices, Sunnyvale, CA). Array-Pro Analyzer Ver4.5 (Media Cybernetics, Silver Spring, MD) was used to determine the signal intensity of each spot and its local background. Scanned images were analyzed using Microarray Data Analysis Tool Ver3.2 software (Filgen).
Signals from Y-27632 treated cells were compared with those from vehicle-treated cells, and genes that showed greater than 3/2-fold change in expression in at least one of the pairwise probe comparisons were considered upregulated, whereas those that showed a change of expression smaller than 2/3-fold were considered downregulated. These analyses were performed three times using TM cells from three different monkeys independently, and genes with common differences in expression among the three experiments were identified as affected genes. The affected genes were further analyzed by gene ontology, in which putative functions of gene products were categorized as "biological process," "cellular component," or "molecular function" by a BLAST homology search of EST sequences available from the National Center for Biotechnology Information.

2.3. Real-Time RT-PCR. Total RNA was isolated from cultured TM cells treated with Y-27632 for $30 \mathrm{~min}$ using NucleoSpin RNA (Macherey-Nagel, Düren, Germany). Total RNA was reverse transcribed (PrimeScript RT Master Mix; Takara Bio Inc., Shiga, Japan) according to the manufacturer's protocol. Quantitative real-time RT-PCR was performed using an ABI Prism 7000 (Life Technologies). Reactions were performed in $20 \mu \mathrm{L}$ of reaction mixture containing $10 \mu \mathrm{L}$ PCR master mix (SYBR Premix Ex Taq II; Takara Bio Inc.), $0.4 \mu \mathrm{M}$ primer pairs, and $2 \mu \mathrm{L} c D N A$ samples. The gene-specific primer pairs were as follows: monkey catalase, forward (F) 5'-GCA AAT CTG TGA GGC CGG GG-3'; reverse (R) 5'-GCG CAT CTA GCA CCG GAG AA-3' and $18 \mathrm{~S}$ ribosomal RNA, (F) 5'-GCC CGA AGC GTT TAC TTT GA-3'; (R) 5'-CCG CGG TCC TAT TCC ATT ATT- $3^{\prime}$. The thermal cycling conditions were $95^{\circ} \mathrm{C}$ for $30 \mathrm{~s}$ and 40 cycles of $95^{\circ} \mathrm{C}$ for $5 \mathrm{~s}$ and $60^{\circ} \mathrm{C}$ for $31 \mathrm{~s}$. All PCR reactions were performed in duplicate.

Relative expression of catalase in the Y-27632-treated samples was compared to that in control samples using the comparative $\mathrm{C}_{\mathrm{T}}$ method $\left(\Delta \Delta \mathrm{C}_{\mathrm{T}}\right.$ method); $18 \mathrm{~S}$ ribosomal RNA was used as an endogenous control. The threshold cycle, $\mathrm{C}_{\mathrm{T}}$, was determined after setting the threshold in the linear amplification phase of the PCR reaction and $\Delta \mathrm{C}_{\mathrm{T}}$ was defined as $\Delta \mathrm{C}_{\mathrm{T}}=\mathrm{C}_{\mathrm{T}}$ (target gene) $-\mathrm{C}_{\mathrm{T}}$ (18S rRNA). Relative expression of the target gene was calculated as: $2^{-\Delta \Delta \mathrm{CT}}, \Delta \Delta \mathrm{C}_{\mathrm{T}}=\Delta \mathrm{C}_{\mathrm{T}}$ (treated sample) $-\Delta \mathrm{C}_{\mathrm{T}}$ (control).

2.4. Intracellular Oxidative Stress Detection. The effects of $\mathrm{Y}-27632$ on the production of ROS were evaluated using CellROX $^{\circledR}$ green reagent (Life Technologies) in the TM cells. These cells were cultured on $6 \mathrm{~cm}$ dishes in DMEM containing $10 \% \mathrm{FBS}$ and antibiotics at $37^{\circ} \mathrm{C}$ in $5 \% \mathrm{CO}_{2}$. After cells had grown to confluence, they were pretreated with Y-27632 for $30 \mathrm{~min}$ and then stimulated with $100 \mu \mathrm{M}$ menadione (Sigma, St. Louis, MO) for $1 \mathrm{~h}$. CellROX reagent was then added to each dish to give a final concentration of $5 \mu \mathrm{M}$ and incubated for $30 \mathrm{~min}$ at $37^{\circ} \mathrm{C}$. After incubation, TM cells were washed in PBS and detached by trypsin/EDTA solution and centrifuged at $1200 \mathrm{rpm}$ for $3 \mathrm{~min}$. The supernatant was removed, and cells were fixed in $4 \%$ paraformaldehyde in PBS for $15 \mathrm{~min}$ and then centrifuged twice at $1200 \mathrm{rpm}$ for $3 \mathrm{~min}$, resuspending in PBS after each 
TABLE 1: Genes that are upregulated in TM cells.

\begin{tabular}{|c|c|c|}
\hline $\begin{array}{l}\text { Accession } \\
\text { number }\end{array}$ & Human RefSeq description & $\begin{array}{c}\text { Fold } \\
\text { change }\end{array}$ \\
\hline DW528016 & gi|75750485|ref|NM_004773.2 Homo sapiens zinc finger, HIT type 3 (ZNHIT3), mRNA & 6.79928 \\
\hline CJ434702 & $\begin{array}{l}\text { gi|20986504|ref|NM_002753.2 Homo sapiens mitogen-activated protein kinase } 10 \text { (MAPK10), } \\
\text { transcript variant 1, mRNA }\end{array}$ & 5.85538 \\
\hline AB168851 & gi|224586874|ref|NM_033124.4 Homo sapiens coiled-coil domain-containing 65 (CCDC65), mRNA & 5.77453 \\
\hline AB169150 & gi|223555972|ref|NR_026827.1 Homo sapiens hypothetical LOC84856 (LOC84856), noncoding RNA & 5.01086 \\
\hline DW523643 & $\begin{array}{l}\text { gi|225903398|ref|NM_001146152.1 Homo sapiens cytochrome P450, family 51, subfamily A, polypeptide } 1 \\
\text { (CYP51A1), transcript variant 2, mRNA }\end{array}$ & 4.6977 \\
\hline AK240630 & gi|4503754|ref|NM_002021.1 Homo sapiens flavin-containing monooxygenase 1 (FMO1), mRNA & 4.64946 \\
\hline BB894083 & gi|154689768|ref|NM_020840.1 Homo sapiens folliculin-interacting protein 2 (FNIP2), mRNA & 4.52052 \\
\hline $\mathrm{AB} 168218$ & gi|85060516|ref|NM_199321.2 Homo sapiens zona pellucida-binding protein 2 (ZPBP2), transcript variant 2, mRNA & 4.20286 \\
\hline AB168199 & $\begin{array}{c}\text { gi|156523965|ref|NM_001102470.1 Homo sapiens alcohol dehydrogenase } 6 \text { (class V) (ADH6), } \\
\text { transcript variant 1, mRNA }\end{array}$ & 3.89514 \\
\hline AB172502 & $\begin{array}{c}\text { gi|50897849|ref|NM_001001936.1 Homo sapiens actin filament-associated protein 1-like } 2 \text { (AFAP1L2), } \\
\text { transcript variant 1, mRNA }\end{array}$ & 3.84241 \\
\hline CJ448047 & $\begin{array}{l}\text { gi|46909588|ref|NM_002731.2 Homo sapiens protein kinase, cAMP-dependent, catalytic, beta (PRKACB), } \\
\text { transcript variant 2, mRNA }\end{array}$ & 3.75324 \\
\hline DC857227 & $\begin{array}{c}\text { gi|239752603|ref|XM_002348257.1 PREDICTED: Homo sapiens similar to immunoglobulin lambda-like } \\
\text { polypeptide } 1 \text { (LOC100294459), mRNA }\end{array}$ & 3.66898 \\
\hline CJ449582 & gi|9506614|ref|NM_019023.1 Homo sapiens protein arginine methyltransferase 7 (PRMT7), mRNA & 3.64058 \\
\hline EF208813 & $\begin{array}{c}\text { gi|24797075|ref|NM_002121.4 Homo sapiens major histocompatibility complex, class II, } \\
\text { DP beta 1 (HLA-DPB1), mRNA }\end{array}$ & 3.57386 \\
\hline DQ417745 & $\begin{array}{c}\text { gi|194,248,050|ref|NM_000839.3 Homo sapiens glutamate receptor, metabotropic } 2 \text { (GRM2), } \\
\text { transcript variant 1, mRNA }\end{array}$ & 3.5509 \\
\hline AB049894 & gi|66571326|ref|NM_020914.3 Homo sapiens ring finger protein 213 (RNF213), mRNA & 3.51068 \\
\hline DC850932 & $\begin{array}{l}\text { gi|117676364|ref|NM_014350.2 Homo sapiens tumor necrosis factor, alpha-induced protein } 8 \text { (TNFAIP8), } \\
\text { transcript variant 1, mRNA }\end{array}$ & 3.47535 \\
\hline BB877436 & gi|142360165|ref|NM_005123.2 Homo sapiens nuclear receptor subfamily 1, group $\mathrm{H}$, member 4 (NR1H4), mRNA & 3.46906 \\
\hline AB174726 & gi|22208962|ref|NM_016150.3 Homo sapiens ankyrin repeat and SOCS box-containing 2 (ASB2), mRNA & 3.30479 \\
\hline AB174122 & $\begin{array}{c}\text { gi|209862773|ref|NM_002483.4 Homo sapiens carcinoembryonic antigen-related cell adhesion molecule } 6 \\
\text { (nonspecific cross-reacting antigen) (CEACAM6), mRNA }\end{array}$ & 3.27904 \\
\hline AB173773 & gi|38569483|ref|NM_017641.2 Homo sapiens kinesin family member 21A (KIF21A), mRNA & 3.26679 \\
\hline DK578446 & gi|15718677|ref|NM_033257.2 Homo sapiens DiGeorge syndrome critical region gene 6 like (DGCR6L), mRNA & 3.26114 \\
\hline AB168950 & gi|38261961|ref|NM_018179.3 Homo sapiens activating transcription factor 7-interacting protein (ATF7IP), mRNA & 3.25607 \\
\hline CЈ488707 & gi|45333915|ref|NM_178456.2 Homo sapiens chromosome 20 open reading frame 85 (C20orf85), mRNA & 3.24156 \\
\hline DC639327 & $\begin{array}{l}\text { gi|154800442|ref|NM_005074.3 Homo sapiens solute carrier family } 17 \text { (sodium phosphate), } \\
\text { member 1 (SLC17A1), mRNA }\end{array}$ & 3.23693 \\
\hline BB895966 & gi|32483409|ref|NM_000583.2 Homo sapiens group-specific component (vitamin D-binding protein) (GC), mRNA & 3.22662 \\
\hline DQ417744 & $\begin{array}{c}\text { gi|194248050|ref|NM_000839.3 Homo sapiens glutamate receptor, metabotropic } 2 \text { (GRM2), } \\
\text { transcript variant 1, mRNA }\end{array}$ & 3.1862 \\
\hline AB168486 & gi|195972893|ref|NM_152764.2 Homo sapiens chromosome 16 open reading frame 73 (C16orf73), mRNA & 3.17552 \\
\hline AB047624 & gi|45446748|ref|NM_004984.2 Homo sapiens kinesin family member 5A (KIF5A), mRNA & 3.1749 \\
\hline CJ446015 & $\begin{array}{c}\text { gi|187761371|ref|NM_004044.5 Homo sapiens 5-aminoimidazole-4-carboxamide ribonucleotide } \\
\text { formyltransferase/IMP cyclohydrolase (ATIC), mRNA }\end{array}$ & 3.17026 \\
\hline AB171508 & gi|89276768|ref|NM_002747.3 Homo sapiens mitogen-activated protein kinase 4 (MAPK4), mRNA & 3.12296 \\
\hline DC639656 & $\begin{array}{l}\text { gi|148596946|ref|NM_001098483.1 Homo sapiens chromosome } 10 \text { open reading frame } 125 \text { (C10orf125), } \\
\text { transcript variant 1, mRNA }\end{array}$ & 3.11887 \\
\hline AB048996 & $\begin{array}{c}\text { gi|211938419|ref|NM_002898.3 Homo sapiens RNA-binding motif, single stranded interacting protein } 2 \\
\text { (RBMS2), mRNA }\end{array}$ & 3.11444 \\
\hline DC630823 & gi|215422360|ref|NM_004786.2 Homo sapiens thioredoxin-like 1 (TXNL1), transcript variant 1, mRNA & 3.09601 \\
\hline AB173283 & gi|197927150|ref|NM_006158.3 Homo sapiens neurofilament, light polypeptide (NEFL), mRNA & 3.09117 \\
\hline DC633065 & gi|34486089|ref|NM_004152.2 Homo sapiens ornithine decarboxylase antizyme 1 (OAZ1), mRNA & 3.0599 \\
\hline
\end{tabular}


TABle 1: Continued.

\begin{tabular}{|c|c|c|}
\hline $\begin{array}{l}\text { Accession } \\
\text { number }\end{array}$ & Human RefSeq description & $\begin{array}{c}\text { Fold } \\
\text { change }\end{array}$ \\
\hline $\mathrm{AB} 174730$ & gi|115387109|ref|NM_017831.3 Homo sapiens ring finger protein 125 (RNF125), mRNA & 3.03611 \\
\hline AB046044 & gi|170650673|ref|NM_000440.2 Homo sapiens phosphodiesterase 6A, cGMP-specific, rod, alpha (PDE6A), mRNA & 3.03568 \\
\hline AB179171 & $\begin{array}{c}\text { gi|82546851|ref|NM_175605.3 Homo sapiens intraflagellar transport } 88 \text { homolog (Chlamydomonas) (IFT88), } \\
\text { transcript variant 1, mRNA }\end{array}$ & 3.029 \\
\hline AB051155 & $\begin{array}{c}\text { gi|35493712|ref|NM_017890.3 Homo sapiens vacuolar protein sorting } 13 \text { homolog B (yeast) (VPS13B), } \\
\text { transcript variant 5, mRNA }\end{array}$ & 3.00764 \\
\hline AB072740 & gi|155029549|ref|NM_178828.4 Homo sapiens chromosome 9 open reading frame 79 (C9orf79), mRNA & 3.00753 \\
\hline BB881371 & gi|162809333|ref|NM_002864.2 Homo sapiens pregnancy-zone protein (PZP), mRNA & 2.99069 \\
\hline CJ463711 & gi|215272411|ref|NM_001142334.1 Homo sapiens ataxin 2-binding protein 1 (A2BP1), transcript variant 6, mRNA & 2.98758 \\
\hline AB170648 & gi|5174424|ref|NM_006052.1 Homo sapiens Down syndrome critical region gene 3 (DSCR3), mRNA & 2.9606 \\
\hline AB220465 & $\begin{array}{c}\text { gi|58331245|ref|NM_000817.2 Homo sapiens glutamate decarboxylase } 1 \text { (brain, } 67 \text { kDa) (GAD1), } \\
\text { transcript variant GAD67, mRNA }\end{array}$ & 2.95173 \\
\hline AB062990 & gi|33149330|ref|NM_022463.3 Homo sapiens nucleore & 2.94787 \\
\hline AB220509 & gi|139394620|ref|NM_006574.3 Homo sapiens chondroitin sulfate proteoglycan 5 (neuroglycan C) (CSPG5), mRNA & 2.92569 \\
\hline AB070086 & gi|22538813|ref|NM_002985.2 Homo sapiens chemokine (C-C motif) ligand 5 (CCL5), mRNA & 2.92446 \\
\hline $\mathrm{AB} 173147$ & gi|194294550|ref|NM_015989.4 Homo sapiens cysteine sulfinic a & 2.91509 \\
\hline AB051133 & gi|28329444|ref|NM_014379.2 Homo sapiens potassium channel, subfamily V, member 1 (KCNV1), m & 2.87085 \\
\hline AB174705 & gi|51477720|ref|NM_001003811.1 Homo sapiens testis-expresse & 2.86731 \\
\hline CJ469703 & gi|169646771|ref|NM_002064.2 Homo sapiens glutaredoxin (thioltrans & 2.85957 \\
\hline AB220438 & $\begin{array}{r}\text { gi|109633045|ref|NM_001042437.1 Homo sapiens ST3 beta-galactosic } \\
\text { transcript variant } 2, \text { mRNA }\end{array}$ & 2.85364 \\
\hline BB881475 & gi|170650673|ref|NM_000440.2 Homo sapiens phosph & 2.84456 \\
\hline $\mathrm{AB} 168610$ & gi|62632749|ref|NM_014616.1 Homo sapier & 2.84449 \\
\hline AB173806 & $\begin{array}{l}\text { gi|134031964|ref|NR_003491.1 Homo sapiens myocardial infarction associated transcript (nonprotein coding) } \\
\text { (MIAT), noncoding RNA }\end{array}$ & 2.82677 \\
\hline AB063045 & gi|190570175|ref|NM_152906.4 Homo sapiens chromosome 22 open reading frame 25 (C22orf25), mRNA & 2.80672 \\
\hline AB168446 & $\begin{array}{c}\text { gi|223278411|ref|NR_026782.1 Homo sapiens chromosome } 1 \text { open reading frame } 175 \text { (C1orf175), transcript } \\
\text { variant 2, transcribed RNA }\end{array}$ & 2.79215 \\
\hline CJ473171 & $\begin{array}{r}\text { gi|215490055|ref|NM_001142434.1 Homo sapiens meningioma } \\
\text { transcript variant } 2,1\end{array}$ & 2.7834 \\
\hline CJ450383 & $\begin{array}{r}\text { gi|83641894|ref|NM_031157.2 Homo sapiens heterogenec } \\
\text { transcript variant } 2\end{array}$ & 2.7832 \\
\hline DW528650 & gi|58430810|ref|NM_148912.2 $\begin{array}{r}\text { Homo so } \\
\text { transcr }\end{array}$ & 2.77238 \\
\hline AB362499 & gi|48255911|ref|NM_012338.3 Homo sapiens tetraspanin 12 (TSPAN12), mRNA & 2.76878 \\
\hline AB173195 & gi|196049386|ref|NM_002198.2 Homo sapiens interferon regulatory factor 1 (IRF1), mRNA & 2.76544 \\
\hline AB168743 & gi|188536107|ref|NM_001127458.1 Homo sapiens cardiolipin synthase 1 (CRLS1), transcript variant 2, mRNA & 2.76042 \\
\hline CJ444006 & gi|157151724|ref|NM_001004333.3 Homo sapiens ribonuclease, RNase K (RNASEK), mRNA & 2.75719 \\
\hline DK583186 & gi|156631002|ref|NM_006913.3 Homo sapiens ring finger protein 5 (RNF5), mRNA & 2.75394 \\
\hline AB168582 & $\begin{array}{l}\text { gi|239757151|ref|XM_002345145.1 PREDICTED: Homo sapiens hypothetical protein LOC100292623 } \\
\text { (LOC100292623), mRNA }\end{array}$ & 2.7528 \\
\hline BB884235 & gi|70906436|ref|NM_000509.4 Homo sapiens fibrinogen gamma chain (FGG), transcript variant gamma-A, mRNA & 2.74831 \\
\hline AB070088 & gi|148613875|ref|NM_144715.3 Homo sapiens EF-hand domain family, member B (EFHB), mRNA & 2.74738 \\
\hline $\mathrm{AB} 174502$ & gi|239757416|ref|XM_002345385.1 PREDICTED: Homo sapiens similar to hCG2019710 (LOC100294049), mRNA & 2.73964 \\
\hline AB172306 & gi|196162714|ref|NM_024786.2 Homo sapiens zinc finger, DHHC-type-containing 11 (ZDHHC11), mRNA & 2.72049 \\
\hline BB878691 & $\begin{array}{c}\text { gi|19743563|ref|NM_000766.3 Homo sapiens cytochrome P450, family 2, subfamily A, polypeptide } 13 \\
\text { (CYP2A13), mRNA }\end{array}$ & 2.72031 \\
\hline AB174483 & gi|55775474|ref|NM_194326.2 Homo sapiens ribosomal protein S19-binding protein 1 (RPS19BP1), mRNA & 2.68579 \\
\hline DC632651 & gi|22538474|ref|NM_018955.2 Homo sapiens ubiquitin B (UBB), mRNA & 2.66418 \\
\hline AB168353 & gi|197927266|ref|NM_004388.2 Homo sapiens chitobiase, di-N-acetyl (CTBS), mRNA & 2.64444 \\
\hline
\end{tabular}


TABle 1: Continued.

\begin{tabular}{|c|c|c|}
\hline $\begin{array}{l}\text { Accession } \\
\text { number }\end{array}$ & Human RefSeq description & $\begin{array}{c}\text { Fold } \\
\text { change }\end{array}$ \\
\hline AB169323 & gi|156616291|ref|NM_018100.3 Homo sapiens EF-hand domain (C-terminal)-containing 1 (EFHC1), mRNA & 2.6433 \\
\hline АВ048961 & gi|209413742|ref|NM_005458.6 Homo sapiens gamma-aminobutyric acid (GABA) B receptor, 2 (GABBR2), mRNA & 2.63945 \\
\hline AB173162 & gi|73747880|ref|NM_015113.3 Homo sapiens zinc finger, ZZ-type with EF-hand domain 1 (ZZEF1), mRNA & 2.63631 \\
\hline AB179192 & gi|237681201|ref|NM_019644.3 Homo sapiens ankyrin repeat domain 7 (ANKRD7), mRNA & 2.62211 \\
\hline CJ469417 & gi|169790802|ref|NM_005271.2 Homo sapiens glutamate dehydrogenase 1 (GLUD1), mRNA & 2.62134 \\
\hline AB172772 & $\begin{array}{l}\text { gi|61835190|ref|NM_006578.3 Homo sapiens guanine nucleotide-binding protein (G protein), beta } 5 \text { (GNB5), } \\
\text { transcript variant 1, mRNA }\end{array}$ & 2.61592 \\
\hline DC857715 & gi|169234652|ref|NM_007360.2 Homo sapiens killer cell lectin-like receptor subfamily K, member 1 (KLRK1), mRNA & 2.6139 \\
\hline AB179131 & $\begin{array}{l}\text { gi|40807356|ref|NM_005094.2 Homo sapiens solute carrier family } 27 \text { (fatty acid transporter), member } 4 \\
\text { (SLC27A4), mRNA }\end{array}$ & 2.61058 \\
\hline DC647811 & gi|92091576|ref|NM_015533.3 Homo sapiens dihydroxyacetone kinase 2 homolog (S. cerevisiae) (DAK), mRNA & 2.60217 \\
\hline AB171456 & $\begin{array}{l}\text { gi|197927256|ref|NM_001134664.1 Homo sapiens sterile alpha motif domain-containing } 13 \text { (SAMD13), } \\
\text { transcript variant 3, mRNA }\end{array}$ & 2.59522 \\
\hline CJ490832 & $\begin{array}{l}\text { gi|169205007|ref|XM_001714899.1 PREDICTED: Homo sapiens hypothetical LOC100131988 } \\
\text { (LOC100131988), mRNA }\end{array}$ & 2.58063 \\
\hline DC635714 & gi|57013275|ref|NM_006082.2 Homo sapiens tubulin, alpha 1b (TUBA1B), mRNA & 2.57848 \\
\hline AB055358 & $\begin{array}{l}\text { gi|225735571|ref|NR_027416.1 Homo sapiens nuclear factor erythroid-derived 2-like } 3 \text { pseudogene } \\
\text { (LOC100272146), noncoding RNA }\end{array}$ & 2.57079 \\
\hline CJ458955 & $\begin{array}{c}\text { gi|197382308|ref|NM_183394.2 Homo sapiens } \mathrm{Ca}^{++} \text {-dependent secretion activator (CADPS), transcript } \\
\text { variant 2, mRNA }\end{array}$ & 2.55777 \\
\hline DC636940 & gi|54112387|ref|NM_001005738.1 Homo sapiens formyl peptide receptor 2 (FPR2), transcript variant 2, mRNA & 2.54881 \\
\hline CJ493104 & gi|56676323|ref|NM_001001552.3 Homo sapiens LEM domain-containing 1 (LEMD1), mRNA & 2.5487 \\
\hline AB172122 & $\begin{array}{l}\text { gi|239754513|ref|XM_001716238.2 PREDICTED: Homo sapiens hypothetical LOC100128081 } \\
\text { (LOC100128081), mRNA }\end{array}$ & 2.54837 \\
\hline CJ469678 & $\begin{array}{c}\text { gi|197304748|ref|NM_032727.3 Homo sapiens internexin neuronal intermediate filament protein, } \\
\text { alpha (INA), mRNA }\end{array}$ & 2.53491 \\
\hline AB070172 & gi|32490571|ref|NM_012307.2 Homo sapiens erythrocyte membrane protein band 4.1-like 3 (EPB41L3), mRNA & 2.52667 \\
\hline DW528013 & $\begin{array}{l}\text { gi|225735571|ref|NR_027416.1 Homo sapiens nuclear factor erythroid-derived 2-like } 3 \text { pseudogene } \\
\text { (LOC100272146), noncoding RNA }\end{array}$ & 2.52479 \\
\hline AB171587 & gi|161169016|ref|NM_001111019.1 Homo sapiens neuron navigator 2 (NAV2), transcript variant 4, mRNA & 2.5209 \\
\hline BB888693 & $\begin{array}{c}\text { gi|49574509|ref|NM_016013.2 Homo sapiens NADH dehydrogenase (ubiquinone) } 1 \text { alpha subcomplex, } \\
\text { assembly factor } 1 \text { (NDUFAF1), mRNA }\end{array}$ & 2.51576 \\
\hline BB897871 & $\begin{array}{c}\text { gi|207113180|ref|NM_001097577.2 Homo sapiens angiogenin, ribonuclease, RNase A family, } 5 \text { (ANG), } \\
\text { transcript variant 2, mRNA }\end{array}$ & 2.50309 \\
\hline AB168153 & gi|222352148|ref|NM_018665.2 Homo sapiens DEAD (Asp-Glu-Ala-Asp) box polypeptide 43 (DDX43), mRNA & 2.50236 \\
\hline AB173823 & $\begin{array}{c}\text { gi|148225856|ref|NM_001097579.1 Homo sapiens G protein-coupled receptor } 34 \text { (GPR34), transcript } \\
\text { variant 4, mRNA }\end{array}$ & 2.49912 \\
\hline AB168907 & gi|194688136|ref|NM_002358.3 Homo sapiens MAD2 mitotic arrest deficient-like 1 (yeast) (MAD2L1), mRNA & 2.49825 \\
\hline DK583616 & gi|18390348|ref|NM_000972.2 Homo sapiens ribosomal protein L7a (RPL7A), mRNA & 2.49444 \\
\hline CJ471599 & gi|197245445|ref|NM_024958.2 Homo sapiens neurensin 2 (NRSN2), mRNA & 2.49281 \\
\hline AB179111 & gi|118572587|ref|NM_001761.2 Homo sapiens cyclin F (CCNF), mRNA & 2.49071 \\
\hline AB060886 & gi|23199979|ref|NM_022470.2 Homo sapiens zinc finger, matrin type 3 (ZMAT3), transcript variant 1, mRNA & 2.48362 \\
\hline AB056810 & $\begin{array}{l}\text { gi|126131101|ref|NM_138694.3 Homo sapiens polycystic kidney and hepatic disease } 1 \text { (autosomal recessive) } \\
\text { (PKHD1), transcript variant 1, mRNA }\end{array}$ & 2.48299 \\
\hline DC645529 & gi|4501988|ref|NM_001134.1 Homo sapiens alpha-fetoprotein (AFP), mRNA & 2.46808 \\
\hline AB050420 & gi|189095267|ref|NM_000554.4 Homo sapiens cone-rod homeobox (CRX), mRNA & 2.45627 \\
\hline AB243403 & gi|116235483|ref|NM_002701.4 Homo sapiens POU class 5 homeobox 1 (POU5F1), transcript variant 1, mRNA & 2.4534 \\
\hline AB173020 & gi|94420687|ref|NM_004233.3 Homo sapiens CD83 molecule (CD83), transcript variant 1, mRNA & 2.44967 \\
\hline DC643036 & gi|50409862|ref|NM_017584.5 Homo sapiens myoinositol oxygenase (MIOX), mRNA & 2.44791 \\
\hline BB897024 & gi|142976728|ref|NM_016245.3 Homo sapiens hydroxysteroid (17-beta) dehydrogenase 11 (HSD17B11), mRNA & 2.43894 \\
\hline DW522619 & gi|34147617|ref|NM_138807.2 Homo sapiens chromosome 3 open reading frame 31 (C3orf31), mRNA & 2.43416 \\
\hline
\end{tabular}


TABle 1: Continued.

\begin{tabular}{|c|c|c|}
\hline $\begin{array}{l}\text { Accession } \\
\text { number }\end{array}$ & Human RefSeq description & $\begin{array}{l}\text { Fold } \\
\text { change }\end{array}$ \\
\hline DC645828 & $\begin{array}{c}\text { gi|16332359|ref|NM_033487.1 Homo sapiens cell division cycle 2-like } 1 \text { (PITSLRE proteins) (CDC2L1), } \\
\text { transcript variant 3, mRNA }\end{array}$ & 2.43092 \\
\hline AB050260 & gi|203098333|ref|NM_032133.4 Homo sapiens MYCBP-associated protein (MYCBPAP), mRNA & 2.41286 \\
\hline CJ436262 & gi|150010638|ref|NM_015276.1 Homo sapiens ubiquitin specific peptidase 22 (USP22), mRNA & 2.39886 \\
\hline AB056381 & $\begin{array}{l}\text { gi|225735571|ref|NR_027416.1 Homo sapiens nuclear factor erythroid-derived 2-like } 3 \text { pseudogene } \\
\text { (LOC100272146), noncoding RNA }\end{array}$ & 2.39471 \\
\hline CJ443349 & $\begin{array}{c}\text { gi }|83367079| \text { ref|NM_003801.3 Homo sapiens glycosylphosphatidylinositol anchor attachment protein } 1 \\
\text { homolog (yeast) (GPAA1), mRNA }\end{array}$ & 2.39333 \\
\hline $\mathrm{AB} 171767$ & $\begin{array}{c}\text { gi|126273571|ref|NM_144586.5 Homo sapiens LY6/PLAUR domain-containing } 1 \text { (LYPD1), transcript } \\
\text { variant 1, mRNA }\end{array}$ & 2.393 \\
\hline $\mathrm{AB} 056817$ & $\begin{array}{c}\text { gi|58535452|ref|NM_001011649.1 Homo sapiens CDK5 regulatory subunit-associated protein 2 (CDK5RAP2), } \\
\text { transcript variant 2, mRNA }\end{array}$ & 2.39285 \\
\hline AB174345 & gi|145208007|ref|NM_173688.2 Homo sapiens $\mathrm{Na}^{+} / \mathrm{K}^{+}$-transporting ATPase interacting 3 (NKAIN3), mRNA & 2.39236 \\
\hline DC648733 & $\begin{array}{c}\text { gi|134133239|ref|NM_032151.4 Homo sapiens pterin-4 alpha-carbinolamine dehydratase/dimerization cofactor } \\
\text { of hepatocyte nuclear factor } 1 \text { alpha (TCF1) } 2 \text { (PCBD2), mRNA }\end{array}$ & 2.39231 \\
\hline CJ435057 & $\begin{array}{l}\text { gi|189083855|ref|NM_000815.4 Homo sapiens gamma-aminobutyric acid (GABA) A receptor, delta } \\
\text { (GABRD), mRNA }\end{array}$ & 2.38598 \\
\hline $\mathrm{AB} 172049$ & gi|41281366|ref|NM_001440.2 Homo sapiens exostoses (multiple)-like 3 (EXTL3), mRNA & 2.37312 \\
\hline CJ489397 & gi|71143136|ref|NM_005342.2 Homo sapiens high-mobility group box 3 (HMGB3), mRNA & 2.36471 \\
\hline AB170096 & gi|42741653|ref|NM_007375.3 Homo sapiens TAR DNA-binding protein (TARDBP), mRNA & 2.36217 \\
\hline AB056391 & gi|169216999|ref|XM_001720515.1 PREDICTED: Homo sapiens similar to pro-pol protein (LOC100129323), mRNA & 2.3496 \\
\hline AB071089 & gi|167900475|ref|NM_001080850.2 Homo sapiens coiled-coil domain-containing 30 (CCDC30), mRNA & 2.34558 \\
\hline DK579603 & gi|84626579|ref|NM_025108.2 Homo sapiens chromosome 16 open reading frame 59 (C16orf59), mRNA & 2.34371 \\
\hline CJ431113 & gi|226246632|ref|NR_027451.1 Homo sapiens hypothetical LOC647979 (LOC647979), noncoding RNA & 2.34265 \\
\hline DK580610 & $\begin{array}{l}\text { gi|34335291|ref|NM_003312.4 Homo sapiens thiosulfate sulfurtransferase (rhodanese) (TST), nuclear gene } \\
\text { encoding mitochondrial protein, mRNA }\end{array}$ & 2.3426 \\
\hline AB168450 & gi|81295815|ref|NM_012337.2 Homo sapiens coiled-coil domain-containing 19 (CCDC19), mRNA & 2.34063 \\
\hline DQ159931 & $\begin{array}{c}\text { gi|163659857|ref|NM_000828.4 Homo sapiens glutamate receptor, ionotrophic, AMPA } 3 \text { (GRIA3), transcript } \\
\text { variant 2, mRNA }\end{array}$ & 2.33939 \\
\hline $\mathrm{AB} 173516$ & gi|36287116|ref|NM_014319.3 Homo sapiens LEM domain-containing 3 (LEMD3), mRNA & 2.33925 \\
\hline $\mathrm{AB} 173575$ & gi|56550100|ref|NM_020978.3 Homo sapiens amylase, alpha 2B (pancreatic) (AMY2B), mRNA & 2.33548 \\
\hline AB169015 & gi|93277104|ref|NM_173812.4 Homo sapiens dpy-19-like 2 (C. elegans) (DPY19L2), mRNA & 2.33006 \\
\hline BB898675 & gi|70906438|ref|NM_021870.2 Homo sapiens fibrinogen gamma chain (FGG), transcript variant gamma-B, mRNA & 2.32385 \\
\hline DK579646 & $\begin{array}{c}\text { gi|153791317|ref|NM_032332.3 Homo sapiens mitogen-activated protein kinase organizer } 1 \text { (MORG1), } \\
\text { transcript variant 2, mRNA }\end{array}$ & 2.32308 \\
\hline AB071115 & gi|111548669|ref|NM_153376.2 Homo sapiens coiled-coil domain-containing 96 (CCDC96), mRNA & 2.31559 \\
\hline DC632824 & $\begin{array}{c}\text { gi|23110926|ref|NM_002799.2 Homo sapiens proteasome (prosome, macropain) subunit, beta type, } 7 \\
\text { (PSMB7), mRNA }\end{array}$ & 2.31501 \\
\hline BB896362 & gi|188595719|ref|NM_005141.3 Homo sapiens fibrinogen beta chain (FGB), mRNA & 2.3131 \\
\hline AB292416 & gi|143770740|ref|NM_001083899.1 Homo sapiens glycoprotein VI (platelet) (GP6), transcript variant 1, mRNA & 2.311 \\
\hline AB055350 & gi|67782353|ref|NM_001024844.1 Homo sapiens CD82 molecule (CD82), transcript variant 2, mRNA & 2.31014 \\
\hline AB168962 & gi|210147405|ref|NM_152621.5 Homo sapiens sphingomyelin synthase 2 (SGMS2), transcript variant 1, mRNA & 2.30806 \\
\hline AB168166 & $\begin{array}{l}\text { gi|156415985|ref|NM_014579.2 Homo sapiens solute carrier family } 39 \text { (zinc transporter), member } 2 \\
\text { (SLC39A2), mRNA }\end{array}$ & 2.30287 \\
\hline AB172981 & $\begin{array}{c}\text { gi|73695942|ref|NM_001010927.2 Homo sapiens T-cell lymphoma invasion and metastasis } 2 \text { (TIAM2), } \\
\text { transcript variant 2, mRNA }\end{array}$ & 2.29903 \\
\hline CJ441025 & gi|153252025|ref|NM_001830.3 Homo sapiens chloride channel 4 (CLCN4), mRNA & 2.29786 \\
\hline CJ445440 & gi|42764686|ref|NM_022652.2 Homo sapiens dual specificity phosphatase 6 (DUSP6), transcript variant 2, mRNA & 2.29536 \\
\hline AB179072 & gi|156119614|ref|NM_006901.2 Homo sapiens myosin IXA (MYO9A), mRNA & 2.28584 \\
\hline AB060229 & $\begin{array}{l}\text { gi|239756940|ref|XM_001718053.2 PREDICTED: Homo sapiens similar to CD300C antigen } \\
\text { (LOC100130520), mRNA }\end{array}$ & 2.28415 \\
\hline
\end{tabular}


Table 1: Continued.

\begin{tabular}{|c|c|c|}
\hline $\begin{array}{l}\text { Accession } \\
\text { number }\end{array}$ & Human RefSeq description & $\begin{array}{c}\text { Fold } \\
\text { change }\end{array}$ \\
\hline CJ480802 & gi|71772428|ref|NM_001021.3 Homo sapiens ribosomal protein S17 (RPS17), mRNA & 2.28212 \\
\hline K581053 & gi|63054873|ref|NM_001615.3 Homo sapiens actin, gamma 2, smooth muscle, enteric (ACTG2), mRNA & 2.27782 \\
\hline AB046030 & gi|169210010|ref|XR_040492.1 PREDICTED: Homo sapiens hypothetical LOC440386 (LOC440386), miscRNA & 2.27371 \\
\hline AB174638 & $\begin{array}{c}\text { gi|44680147|ref|NM_203327.1 Homo sapiens solute carrier family } 23 \text { (nucleobase transporters), } \\
\text { member } 2 \text { (SLC23A2), transcript variant 2, mRNA }\end{array}$ & 2.2635 \\
\hline CJ469779 & gi|95147340|ref|NM_004603.2 Homo sapiens syntaxin 1A (brain) (STX1A), mRNA & 2.25959 \\
\hline DC632108 & gi|225637497|ref|NR_003286.2 Homo sapiens 18S ribosomal RNA (LOC100008588), noncoding RNA & 2.25815 \\
\hline AB170956 & $\begin{array}{l}\text { gi|65506441|ref|NM_000282.2 Homo sapiens propionyl coenzyme A carboxylase, alpha polypeptide (PCCA), } \\
\text { nuclear gene encoding mitochondrial protein, transcript variant } 1 \text {, mRNA }\end{array}$ & 2.25785 \\
\hline DC858184 & $\begin{array}{l}\text { gi|13569959|ref|NM_030980.1 Homo sapiens interferon stimulated exonuclease gene } 20 \text { kDa-like } 2 \\
\text { (ISG20L2), mRNA }\end{array}$ & 2.25755 \\
\hline CЈ470090 & $\begin{array}{c}\text { gi|169790838|ref|NM_004172.4 Homo sapiens solute carrier family } 1 \text { (glial high affinity glutamate transporter), } \\
\text { member } 3 \text { (SLC1A3), mRNA }\end{array}$ & 2.24996 \\
\hline DW522847 & $\begin{array}{c}\text { gi|215983055|ref|NM_031471.5 Homo sapiens fermitin family homolog } 3 \text { (Drosophila) (FERMT3), transcript } \\
\text { variant URP2SF, mRNA }\end{array}$ & 2.23483 \\
\hline DK577957 & gi|186910295|ref|NM_001126102.1 Homo sapiens haptoglobin (HP), transcript variant 2, mRNA & 2.23107 \\
\hline AB168531 & gi|162951880|ref|NM_001112707.1 Homo sapiens tousled-like kinase 2 (TLK2), transcript variant B, mRNA & 2.22872 \\
\hline AB047603 & gi|149363673|ref|NM_012194.1 Homo sapiens chromosome 11 open reading frame 41 (C11orf41), mRNA & 22681 \\
\hline С642489 & $\begin{array}{c}\text { gi|66392201|ref|NM_002512.2 Homo sapiens nonmetastatic cells 2, protein (NM23B) expressed in (NME2), } \\
\text { transcript variant 1, mRNA }\end{array}$ & 2.22176 \\
\hline B072760 & gi|91754184|ref|NM_152763.3 Homo sapiens chromosome 1 open reading frame 62 (C1orf62), mRNA & 2.2183 \\
\hline AB168708 & gi|36287109|ref|NM_194429.1 Homo sapiens FGFR1 oncogene partner (FGFR1OP), transcript variant 2, mRNA & 2.21393 \\
\hline AB168136 & $\begin{array}{r}\text { gi|93004101|ref|NM_005730.3 Homo sapiens CTD (c } \\
\text { small phosphat }\end{array}$ & 2.20887 \\
\hline DC641033 & $\begin{array}{l}\text { gi|108389126|ref|NM_001042353.1 Homo sapiens family with sequence similarity 110, member A (FAM110A), } \\
\text { transcript variant 3, mRNA }\end{array}$ & 2.20687 \\
\hline CJ453010 & $\begin{array}{r}\text { gi|19743893|ref|NM_133480.1 Homo sapiens transcripti } \\
\text { transcript vari }\end{array}$ & 2.20608 \\
\hline AB168419 & gi|32996736|ref|NM_173083.2 Homo sapiens lin-9 homolog (C. elegans) (LIN9), mRNA & 2.20292 \\
\hline AB169251 & $\begin{array}{l}\text { gi|148727318|ref|NM_001098529.1 Homo sapiens thioredoxin domain-containing } 2 \text { (spermatozoa) (TXNDC2), } \\
\text { transcript variant 2, mRNA }\end{array}$ & 2.2019 \\
\hline B173492 & gi|31542657|ref|NM_018099.3 Homo sap & 2.20171 \\
\hline CJ443285 & gi|62955828|ref|NM_033428.1 Homo sapiens chromosome 9 open reading frame 123 (C9orf123), mRNA & 2.19894 \\
\hline AB169808 & $\begin{array}{r}\text { gi|20336295|ref|NM_018380.2 Homo sapiens DEAD (Asp-Glu-Al } \\
\text { nuclear gene encoding mitochondrial pro }\end{array}$ & 2.1907 \\
\hline AB169213 & $\begin{array}{l}\text { gi|125625349|ref|NM_001790.3 Homo sapiens cell division cycle } 25 \text { homolog C (S. pombe) (CDC25C), } \\
\text { transcript variant 1, mRNA }\end{array}$ & 2.18403 \\
\hline CJ491693 & gi|21361889|ref|NM_021633.2 Homo sapiens Kelch-like 12 (Drosophila) (KLHL12), mRNA & 2.1801 \\
\hline BB876451 & $\begin{array}{l}\text { gi|109389366|ref|NM_000312.2 Homo sapiens protein C (inactivator of coagulation factors Va and VIIIa) } \\
\text { (PROC), mRNA }\end{array}$ & 2.17421 \\
\hline AB171871 & $\begin{array}{l}\text { gi|153070253|ref|NM_001099680.1 Homo sapiens MAGI family member, X-linked (MAGIX), transcript } \\
\text { variant 2, mRNA }\end{array}$ & 2.17287 \\
\hline AB173097 & gi|198041927|ref|NM_139241.2 Homo sapiens FYVE, RhoGEF and PH domain-containing 4 (FGD4), mRNA & 2.16791 \\
\hline AB046632 & gi|136255215|ref|NM_207351.3 Homo sapiens proline-rich transmembrane protein 3 (PRRT3), mRNA & 2.16598 \\
\hline DK577943 & gi|84872083|ref|NR_002798.1 Homo sapiens napsin B aspartic peptidase pseudogene (NAPSB), noncoding RNA & 2.16578 \\
\hline AB168792 & gi|141802709|ref|NM_145263.2 Homo sapiens spermatogenesis associated 18 homolog (rat) (SPATA18), mRNA & 2.16491 \\
\hline BB881148 & $\begin{array}{l}\text { gi|189458840|ref|NM_005942.3 Homo sapiens molybdenum cofactor synthesis } 1 \text { (MOCS1), transcript } \\
\text { variant 2, mRNA }\end{array}$ & 2.16393 \\
\hline CJ472360 & $\begin{array}{c}\text { gi|24497456|ref|NM_139136.2 Homo sapiens potassium voltage-gated channel, Shaw-related subfamily, } \\
\text { member } 2 \text { (KCNC2), transcript variant 1, mRNA }\end{array}$ & 2.15994 \\
\hline CJ430900 & gi|94721262|ref|NM_001040446.1 Homo sapiens myotubularin-related protein 12 (MTMR12), mRNA & 2.15957 \\
\hline
\end{tabular}


TABle 1: Continued.

\begin{tabular}{|c|c|c|}
\hline $\begin{array}{l}\text { Accession } \\
\text { number }\end{array}$ & Human RefSeq description & $\begin{array}{l}\text { Fold } \\
\text { change }\end{array}$ \\
\hline $\mathrm{AB} 171550$ & $\begin{array}{l}\text { gi|19913413|ref|NM_014203.2 Homo sapiens adaptor-related protein complex 2, alpha } 1 \text { subunit (AP2A1), } \\
\text { transcript variant 1, mRNA }\end{array}$ & 2.1589 \\
\hline AB173954 & gi|188595678|ref|NM_014959.2 Homo sapiens caspase recruitment domain family, member 8 (CARD8), mRNA & 2.13719 \\
\hline AB071125 & $\begin{array}{c}\text { gi|89903024|ref|NM_001031735.2 Homo sapiens chromosome } 19 \text { open reading frame } 36 \text { (C19orf36), } \\
\text { transcript variant 1, mRNA }\end{array}$ & 2.13665 \\
\hline AB063014 & gi|170650671|ref|NM_001122769.1 Homo sapiens Leber congenital amaurosis 5 (LCA5), transcript variant 2, mRNA & 2.13524 \\
\hline DC631520 & $\begin{array}{c}\text { gi|189163527|ref|NM_001127700.1 Homo sapiens serpin peptidase inhibitor, clade A (alpha-1 antiproteinase, } \\
\text { antitrypsin), member } 1 \text { (SERPINA1), transcript variant 4, mRNA }\end{array}$ & 2.1306 \\
\hline AY742821 & $\begin{array}{c}\text { gi|59806358|ref|NM_006011.3 Homo sapiens ST8 alpha-N-acetyl-neuraminide alpha-2,8-sialyltransferase } 2 \\
\text { (ST8SIA2), mRNA }\end{array}$ & 2.12885 \\
\hline AK240628 & $\begin{array}{c}\text { gi|160298141|ref|NM_000668.4 Homo sapiens alcohol dehydrogenase 1B (class I), beta polypeptide } \\
\text { (ADH1B), mRNA }\end{array}$ & 2.12458 \\
\hline AB174195 & gi|30794215|ref|NM_030961.1 Homo sapiens tripartite motif-containing 56 (TRIM56), mRNA & 2.12446 \\
\hline DC646861 & $\begin{array}{r}\text { gi|91807120|ref|NM_033087.3 Homo sapiens asparagine-lin } \\
\text { homolog (S. cerevisiae) (ALG2), tr }\end{array}$ & 2.12291 \\
\hline AY650365 & gi|27436932|ref|NM_172337.1 Homo sapiens orthodenticle homeobox 2 (OTX2), transcript variant 2, mRNA & 2.11514 \\
\hline DW527197 & gi|219555668|ref|NM_052855.3 Homo sapiens ankyrin repeat domain 40 (ANKRD40), mRNA & 2.115 \\
\hline AB171287 & gi|188497721|ref|NM_001127385.1 Homo sapiens cortexin 3 (CTXN3), transcript variant 2, mRNA & 2.11438 \\
\hline AB173764 & gi|219879811|ref|NM_005475.2 Homo sapiens SH2B adaptor protein 3 (SH2B3), mRNA & 2.10791 \\
\hline DK582787 & gi|221316657|ref|NM_004811.2 Homo sapiens leupaxin (LPXN), transcript variant 2, mRNA & 2.10555 \\
\hline AB070128 & gi|217416373|ref|NM_145038.2 Homo sapiens chromosome 2 open reading frame 39 (C2orf39), mRNA & 2.10225 \\
\hline AB070165 & gi|226491198|ref|NM_182496.2 Homo sapiens coiled-coil domain-containing 38 (CCDC38), mRNA & 2.10075 \\
\hline DK577398 & $\begin{array}{c}\text { gi|52426772|ref|NM_002122.3 Homo sapiens major histocompatibility complex, class II, DQ alpha } 1 \\
\text { (HLA-DQA1), mRNA }\end{array}$ & 2.09004 \\
\hline AB169904 & gi|34147601|ref|NM_004309.3 Homo sapiens rho GI & 2.08484 \\
\hline AB220503 & $\begin{array}{r}\text { gi|237681178|ref|NM_001160260.1 Homo sapiens cat } \\
\text { variant } 6, \mathrm{n}\end{array}$ & 2.08434 \\
\hline $\mathrm{AB} 173401$ & $\begin{array}{r}\text { gi|239750034|ref|XR_039406.2 PREDICTED: Homo s } \\
\text { (LOC100132562), } \mathrm{n}\end{array}$ & 2.08407 \\
\hline $\mathrm{AB} 171785$ & $\begin{array}{c}\text { gi|253970447|ref|NM_014253.3 Homo sapiens odz, odd Oz/ten-m homolog 1(Drosophila) (ODZ1), transcript } \\
\text { variant 3, mRNA }\end{array}$ & 2.08184 \\
\hline AB171491 & $\begin{array}{r}\text { gi|117938287|ref|NM_004171.3 Homo sapiens solute car } \\
\text { member } 2 \text { (SLC }\end{array}$ & 2.08087 \\
\hline $\mathrm{AB} 174571$ & $\begin{array}{l}\text { gi|182765446|ref|NM_001031711.2 Homo sapiens endoplasmic reticulum-Golgi intermediate compartment } \\
\text { (ERGIC) } 1 \text { (ERGIC1), mRNA }\end{array}$ & 2.07886 \\
\hline AB063092 & gi|34577113|ref|NM_015576.1 Homo sapiens ELKS/RAB6-interacting/CAST family member 2 (ERC2), mRNA & 2.07171 \\
\hline AB056378 & gi|189163523|ref|NM_033064.4 Homo sapiens ataxia, cerebellar, Cayman type (ATCAY), mRNA & 2.06758 \\
\hline AB055299 & $\begin{array}{c}\text { gi|163644324|ref|NM_001112732.1 Homo sapiens MCF.2 cell line derived transforming sequence-like (MCF2L), } \\
\text { transcript variant 1, mRNA }\end{array}$ & 2.06139 \\
\hline $\mathrm{AB} 172748$ & gi|119220563|ref|NM_004852.2 Homo sapiens one cut homeobox 2 (ONECUT2), mRNA & 2.05909 \\
\hline $\mathrm{AB} 172478$ & gi|239746981|ref|XR_078603.1 PREDICTED: Homo sapiens similar to putative p150 (LOC100288106), miscRNA & 2.05792 \\
\hline AB170807 & gi|236459850|ref|NM_173569.3 Homo sapiens ubinuclein 2 (UBN2), mRNA & 2.05471 \\
\hline EF208824 & $\begin{array}{c}\text { gi|239740919|ref|XM_002344047.1 PREDICTED: Homo sapiens similar to major histocompatibility complex, } \\
\text { class II, DQ beta 1, transcript variant } 2 \text { (LOC100294318), mRNA }\end{array}$ & 2.05031 \\
\hline AB169481 & $\begin{array}{c}\text { gi|150417992|ref|NM_033312.2 Homo sapiens CDC14 cell division cycle } 14 \text { homolog A (S. cerevisiae) (CDC14A), } \\
\text { transcript variant 2, mRNA }\end{array}$ & 2.04992 \\
\hline $\mathrm{AB} 171520$ & gi|56243494|ref|NM_004586.2 Homo sapiens ribosomal protein S6 kinase, $90 \mathrm{kDa}$, polypeptide 3 (RPS6KA3), mRNA & 2.0465 \\
\hline DC629151 & gi|215982788|ref|NM_000477.5 Homo sapiens albumin (ALB), mRNA & 2.04345 \\
\hline DC640591 & $\begin{array}{c}\text { gi|208609965|ref|NM_001135664.1 Homo sapiens RAB7, member RAS oncogene family-like 1 (RAB7L1), } \\
\text { transcript variant 4, mRNA }\end{array}$ & 2.04167 \\
\hline BB887273 & gi|215,982,788|ref|NM_000477.5 Homo sapiens albumin (ALB), mRNA & 2.0414 \\
\hline
\end{tabular}


Table 1: Continued.

\begin{tabular}{|c|c|c|}
\hline $\begin{array}{l}\text { Accession } \\
\text { number }\end{array}$ & Human RefSeq description & $\begin{array}{c}\text { Fold } \\
\text { change }\end{array}$ \\
\hline CJ435276 & $\begin{array}{l}\text { gi|75,812,975|ref|NM_001033574.1 Homo sapiens archaelysin family metallopeptidase } 2 \text { (AMZ2), transcript } \\
\text { variant 6, mRNA }\end{array}$ & 2.04064 \\
\hline DC643114 & $\begin{array}{l}\text { gi }|33519462| \text { ref|NM_004544.2 Homo sapiens NADH dehydrogenase (ubiquinone) } 1 \text { alpha subcomplex, 10, } 42 \mathrm{kDa} \\
\text { (NDUFA10), nuclear gene encoding mitochondrial protein, mRNA }\end{array}$ & 2.03575 \\
\hline CJ436048 & $\begin{array}{c}\text { gi|62865867|ref|NM_004102.3 Homo sapiens fatty acid-binding protein 3, muscle and heart } \\
\text { (mammary-derived growth inhibitor) (FABP3), mRNA }\end{array}$ & 2.03528 \\
\hline AB179303 & $\begin{array}{c}\text { gi|196162694|ref|NM_003401.3 Homo sapiens X-ray repair complementing defective repair in Chinese hamster } \\
\text { cells } 4 \text { (XRCC4), transcript variant 1, mRNA }\end{array}$ & 2.03322 \\
\hline AB171313 & gi|146219840|ref|NM_020709.1 Homo sapiens PNMA-like 2 (PNMAL2), mRNA & 2.02925 \\
\hline AB173369 & gi|149363694|ref|NM_001009984.1 Homo sapiens chromosome 20 open reading frame 194 (C20orf194), mRNA & 2.01079 \\
\hline $\mathrm{AB} 171481$ & gi|18496982|ref|NM_015526.1 Homo sapiens CAP-GLY domain-containing linker protein 3 (CLIP3), mRNA & 2.00151 \\
\hline AB174068 & gi|84872123|ref|NR_002833.1 Homo sapiens dpy-19-like 2 pseudogene 1 (C. elegans) (DPY19L2P1), noncoding RNA & 1.99958 \\
\hline AB050434 & gi|239753181|ref|XM_002345525.1 PREDICTED: Homo sapiens similar to hCG2041348 (LOC100293610), mRNA & 1.99905 \\
\hline DK577438 & gi|88999575|ref|NM_002622.4 Homo sapiens prefoldin subunit 1 (PFDN1), mRNA & 1.99519 \\
\hline AB172315 & $\begin{array}{l}\text { gi|239753426|ref|XR_038411.2 PREDICTED: Homo sapiens similar to eukaryotic translation elongation factor } 1 \text { beta } \\
\text { 2(LOC646973), miscRNA }\end{array}$ & 1.99372 \\
\hline BB895222 & gi|38372939|ref|NM_001185.2 Homo sapiens alpha-2-glycoprotein 1, zinc-binding (AZGP1), mRNA & 1.99214 \\
\hline AB173728 & $\begin{array}{l}\text { gi|111154086|ref|NM_020631.3 Homo sapiens pleckstrin homology domain-containing, family G } \\
\text { (with RhoGef domain) member } 5 \text { (PLEKHG5), transcript variant 1, mRNA }\end{array}$ & 1.98959 \\
\hline DC647709 & gi|28416926|ref|NM_002560.2 Homo sapiens purinergic receptor P2X, ligand-gated ion channel, 4 (P2RX4), mRNA & 1.98692 \\
\hline AB048919 & gi|156766083|ref|NM_031418.2 Homo sapiens anoctamin 3 (ANO3), mRNA & 1.98684 \\
\hline AB179103 & gi|18860913|ref|NM_021818.2 Homo sapiens salvador homolog 1 (Drosophila) (SAV1), mRNA & 1.97681 \\
\hline AB046073 & gi|118498342|ref|NM_014861.2 Homo sapiens ATPase, $\mathrm{Ca}^{++}$transporting, type 2C, member 2 (ATP2C2), mRNA & 1.97604 \\
\hline $\mathrm{AB} 172144$ & gi|190341103|ref|NM_015163.5 Homo sapiens tripartite motif-containing 9 (TRIM9), transcript variant 1, mRNA & 1.97599 \\
\hline DK583369 & gi|171460955|ref|NM_005800.4 Homo sapiens ubiquitin-specific peptidase like 1 (USPL1), mRNA & 1.9752 \\
\hline $\mathrm{AB} 174098$ & gi|110815799|ref|NM_024345.3 Homo sapiens DDB1 and CUL4-associated factor 10 (DCAF10), mRNA & 1.97309 \\
\hline DW526268 & gi|31083173|ref|NM_181078.1 Homo sapiens interleukin 21 receptor (IL21R), transcript variant 2, mRNA & 1.97258 \\
\hline AB171701 & gi|20544144|ref|NM_139062.1 Homo sapiens casein kinase 1, delta (CSNK1D), transcript variant 2, mRNA & 1.96537 \\
\hline CJ493302 & gi|17136150|ref|NM_004724.2 Homo sapiens ZW10, kinetochore associated, homolog (Drosophila) (ZW10), mRNA & 1.96534 \\
\hline DK577545 & $\begin{array}{l}\text { gi|24797075|ref|NM_002121.4 Homo sapiens major histocompatibility complex, class II, DP beta } 1 \\
\text { (HLA-DPB1), mRNA }\end{array}$ & 1.96346 \\
\hline CJ458429 & gi|71067335|ref|NM_031462.2 Homo sapiens CD99 molecule-like 2 (CD99L2), transcript variant 1, mRNA & 1.96208 \\
\hline AB172752 & gi|32698785|ref|NM_182490.1 Homo sapiens zinc finger protein 227 (ZNF227), mRNA & 1.95671 \\
\hline AB171668 & gi|40385866|ref|NM_199227.1 Homo sapiens methionine aminopeptidase 1D (MAP1D), mRNA & 1.95399 \\
\hline AB051117 & gi|82617625|ref|NM_001037293.1 Homo sapiens paralemmin 2 (PALM2), transcript variant 2, mRNA & 1.9528 \\
\hline AB169059 & gi|91992151|ref|NM_000616.3 Homo sapiens CD4 molecule (CD4), mRNA & 1.95226 \\
\hline CJ443230 & $\begin{array}{c}\text { gi|46094085|ref|NM_022758.4 Homo sapiens chromosome } 6 \text { open reading frame } 106 \text { (C6orf106), transcript } \\
\text { variant 2, mRNA }\end{array}$ & 1.95082 \\
\hline AB178987 & gi|38045951|ref|NM_021030.2 Homo sapiens zinc finger protein 14 (ZNF14), mRNA & 1.94816 \\
\hline $\mathrm{AB} 172387$ & gi|163659919|ref|NM_052839.3 Homo sapiens pannexin 2 (PANX2), transcript variant 1, mRNA & 1.94536 \\
\hline $\mathrm{AB} 168775$ & $\begin{array}{l}\text { gi|223468671|ref|NM_001145135.1 Homo sapiens carnitine palmitoyltransferase 1B (muscle) (CPT1B), } \\
\text { nuclear gene encoding mitochondrial protein, transcript variant 6, mRNA }\end{array}$ & 1.94062 \\
\hline DC633198 & $\begin{array}{c}\text { gi|206725531|ref|NM_001826.2 Homo sapiens CDC28 protein kinase regulatory subunit 1B (CKS1B), } \\
\text { transcript variant 1, mRNA }\end{array}$ & 1.93773 \\
\hline $\mathrm{AB} 172044$ & $\begin{array}{l}\text { gi|25168266|ref|NM_170709.1 Homo sapiens serum/glucocorticoid-regulated kinase family, member } 3 \text { (SGK3), } \\
\text { transcript variant 2, mRNA }\end{array}$ & 1.93543 \\
\hline DK578185 & $\begin{array}{l}\text { gi|239754745|ref|XM_002346052.1 PREDICTED: Homo sapiens hypothetical protein LOC100293771 } \\
\text { (LOC100293771), mRNA }\end{array}$ & 1.93421 \\
\hline AB171597 & $\begin{array}{l}\text { gi|113951732|ref|NM_012095.4 Homo sapiens adaptor-related protein complex 3, mu } 1 \text { subunit (AP3M1), } \\
\text { transcript variant 2, mRNA }\end{array}$ & 1.93313 \\
\hline AB179405 & gi|31543301|ref|NM_032600.2 Homo sapiens coiled-coil domain-containing 54 (CCDC54), mRNA & 1.93038 \\
\hline
\end{tabular}


TABle 1: Continued.

\begin{tabular}{|c|c|c|}
\hline $\begin{array}{l}\text { Accession } \\
\text { number }\end{array}$ & Human RefSeq description & $\begin{array}{l}\text { Fold } \\
\text { change }\end{array}$ \\
\hline AB179267 & gi|37594443|ref|NM_015896.2 Homo sapiens zinc finger, MYND-type-containing 10 (ZMYND10), mRNA & 1.92859 \\
\hline DC640525 & gi|35493837|ref|NM_004902.2 Homo sapiens RNA-binding motif protein 39 (RBM39), transcript variant 2, mRNA & 1.92337 \\
\hline АB049869 & gi|239753181|ref|XM_002345525.1 PREDICTED: Homo sapiens similar to hCG2041348 (LOC100293610), mRNA & 1.92159 \\
\hline DW528250 & gi|52487034|ref|NM_004618.3 Homo sapiens topoisomerase (DNA) III alpha (TOP3A), mRNA & 1.91924 \\
\hline DC636463 & gi|78214521|ref|NM_001035258.1 Homo sapiens ribosomal protein L38 (RPL38), transcript variant 2, mRNA & 1.91826 \\
\hline AB179052 & $\begin{array}{c}\text { gi|115511031|ref|NM_004432.2 Homo sapiens ELAV- (embryonic lethal, abnormal vision, Drosophila-) like } 2 \\
\text { (Hu antigen B) (ELAVL2), mRNA }\end{array}$ & 1.91696 \\
\hline AB168809 & $\begin{array}{r}\text { gi|37622352|ref|NM_003551.2 Homo sapiens nonmetastatic ce } \\
\text { kinase (NME5), n }\end{array}$ & 1.91336 \\
\hline CJ435007 & gi|115527063|ref|NM_004859.3 Homo sapiens clathri & 1.91318 \\
\hline AB171499 & gi|50845406|ref|NM_031444.2 Homo sapiens chromosome 22 open reading frame 13 (C22orf13), mRNA & 1.91088 \\
\hline DC647333 & gi|118600974|ref|NM_007269.2 Homo sapiens syntaxin-binding protein 3 (STXBP3), mRNA & 1.90575 \\
\hline $\mathrm{AB} 172403$ & gi|142976637|ref|NM_017420.3 Homo sapiens SIX homeobox 4 (SIX4), mRNA & 1.89362 \\
\hline $\mathrm{AB} 174282$ & gi|31543080|ref|NM_016210.2 Homo sapiens chromosome 3 open reading frame 18 (C3orf18), mRNA & 1.89271 \\
\hline DC648759 & gi|6382072|ref|NM_005258.2 Homo sapiens GTP cyclohydrolase I feedback regulator (GCHFR), mRNA & 1.88951 \\
\hline AB169033 & $\begin{array}{c}\text { gi|195927038|ref|NM_001786.3 Homo sapiens cell division cycle 2, G1 to S and G2 to M (CDC2), transcript } \\
\text { variant } 1 \text {, mRNA }\end{array}$ & 1.88515 \\
\hline AB173309 & gi|209447072|ref|NM_001135806.1 Homo sap & \\
\hline & & \\
\hline & gi|86787650|ref|NM_014800.9 Homo sapiens engulfme & 7945 \\
\hline & gi|209364624|ref|NM_001822.4 Homo sapiens chin & 7132 \\
\hline $\mathrm{AB} 171236$ & $\begin{array}{r}\text { gi|19743893|ref|NM_133480.1 Homo sapiens transcripti } \\
\text { transcript vari }\end{array}$ & 1.86897 \\
\hline BB885210 & gi|32484974|ref|NM_006721.2 Homo sapiens adeno & 1.8686 \\
\hline AB169067 & gi|188528615|ref|NM_182911.3 Homo sapiens tes & 1.86706 \\
\hline CJ464698 & $\begin{array}{r}\text { gi|221307560|ref|NR_026669.1 Homo sapiens synapt } \\
\text { transcript var }\end{array}$ & 1.86542 \\
\hline AB179482 & gi|51173716|ref|NM_006720.3 Homo sapiens actin-binding LIM protein 1 & 1.85972 \\
\hline CJ442615 & $\begin{array}{c}\text { gi|239745120|ref|XR_015162.2 PREDICTED: Homo sapiens hyp } \\
\text { (LOC727880), miscRNA }\end{array}$ & 1.85021 \\
\hline & gi|170650722|ref|NM_014236.3 Homo sapiens & 1.8499 \\
\hline AY650307 & gi|51599155|ref|NM_001273.2 Homo sapiens chromodor & 1.84509 \\
\hline DW525872 & $\begin{array}{r}\text { gi|77404354|ref|NM_003908.3 Homo sapiens eukaryotic } \\
\text { (EIF2S2), }\end{array}$ & 1.84501 \\
\hline & gi|78190459|ref|NM_000978.3 Homo sap & 1.8440 \\
\hline $\mathrm{AB} 174451$ & $\begin{array}{c}\text { gi|223941821|ref|NM_014342.3 Homo sapiens mitochondrial carrier homolog } 2 \text { (C. elegans) (MTCH2), } \\
\text { nuclear gene encoding mitochondrial protein, mRNA }\end{array}$ & 1.84017 \\
\hline AB066534 & gi|188528627|ref|NM_033109.3 Homo sapiens polyribonucleotide nucleotidyltransferase 1 (PNPT1), mRNA & 1.83655 \\
\hline BB888999 & gi|160298191|ref|NM_000507.3 Homo sapiens fructose-1,6-bisphosphatase 1 (FBP1), transcript variant 1, mRNA & 1.8352 \\
\hline DC637318 & gi|209977038|ref|NM_016074.3 Homo sapiens bolA homolog 1 (E. coli) (BOLA1), mRNA & 1.83477 \\
\hline AB169205 & $\begin{array}{c}\text { gi|109948303|ref|NM_018225.2 Homo sapiens smu-1 suppressor of mec-8 and unc-52 homolog (C. elegans) } \\
\text { (SMU1), mRNA }\end{array}$ & 1.82627 \\
\hline & gi|19913444|ref|NM_016257.2 Homo sapiens hippocalcin-like 4 (HPCAL4), mRNA & 1.8159 \\
\hline AY650384 & gi|141803509|ref|NM_058164.2 Homo sapiens olfactomedin 2 (OLFM2), mRNA & 1.81587 \\
\hline DC647305 & gi|38372918|ref|NM_001728.2 Homo sapiens basigin (Ok blood group) (BSG), transcript variant 1, mRNA & 1.8093 \\
\hline $\mathrm{AB} 172260$ & $\begin{array}{c}\text { gi|112382251|ref|NM_178313.2 Homo sapiens spectrin, beta, nonerythrocytic } 1 \text { (SPTBN1), } \\
\text { transcript variant 2, mRNA }\end{array}$ & 1.8074 \\
\hline & gi|194097340|ref|NM_002616.2 Homo sapiens period homolog 1 (Drosophila) (PER1), mRNA & 1.80415 \\
\hline & gi|242247096|ref|NM_001340.3 Homo sapiens cylicin, basic protein of sperm head cytoskeleton 2 (CYLC2), mRNA & 1.80079 \\
\hline AB173856 & gi|60302919|ref|NM_001752.2 Homo sapiens catalase (CAT), mRNA & 1.79676 \\
\hline
\end{tabular}


TABle 1: Continued.

\begin{tabular}{|c|c|c|}
\hline $\begin{array}{l}\text { Accession } \\
\text { number }\end{array}$ & Human RefSeq description & $\begin{array}{l}\text { Fold } \\
\text { change }\end{array}$ \\
\hline AB060862 & gi|221219051|ref|NM_031924.4 Homo sapiens radial spoke 3 homolog (Chlamydomonas) (RSPH3), mRNA & 1.79563 \\
\hline CJ470793 & $\begin{array}{c}\text { gi|224586819|ref|NR_027265.1 Homo sapiens Golgi apparatus protein } 1 \text { (GLG1), transcript variant 5, } \\
\text { transcribed RNA }\end{array}$ & 1.79405 \\
\hline DW528583 & $\begin{array}{l}\text { gi|239787833|ref|NM_015139.2 Homo sapiens solute carrier family } 35 \text { (UDP-glucuronic acid/ } \\
\text { UDP-N-acetylgalactosamine dual transporter), member D1 (SLC35D1), mRNA }\end{array}$ & 1.79359 \\
\hline DK580881 & gi|194394144|ref|NM_145870.2 Homo sapiens glutathione transferase zeta 1 (GSTZ1), transcript variant 1, mRNA & 1.792 \\
\hline AB173997 & gi|225543100|ref|NR_027378.1 Homo sapiens hypothetical LOC643763 (LOC643763), noncoding RNA & 1.79131 \\
\hline AY650356 & gi|223718142|ref|NM_173054.2 Homo sapiens reelin (RELN), transcript variant 2, mRNA & 1.78729 \\
\hline DK584117 & gi|15967154|ref|NM_016558.2 Homo sapiens SCAN domain-containing 1 (SCAND1), transcript variant 1, mRNA & 1.78008 \\
\hline DC621384 & gi|15431296|ref|NM_000977.2 Homo sapiens ribosomal protein L13 (RPL13), transcript variant 1, mRNA & 1.77763 \\
\hline DK577712 & gi|109148541|ref|NM_001605.2 Homo sapiens alanyl-tRNA synthetase (AARS), mRNA & 1.77723 \\
\hline $\mathrm{AB} 174251$ & $\begin{array}{l}\text { gi|253314435|ref|NR_027995.1 Homo sapiens ankyrin repeat domain } 20 \text { family, member A2 pseudogene } \\
\text { (LOC284232), noncoding RNA }\end{array}$ & 1.77027 \\
\hline $\mathrm{AB} 174247$ & gi|50897295|ref|NM_001002923.1 Homo sapiens IGF-like family member 4 (IGFL4), mRNA & 1.76977 \\
\hline CJ490195 & gi|78190459|ref|NM_000978.3 Homo sapiens ribosomal protein L23 (RPL23), mRNA & 1.76786 \\
\hline $\mathrm{AB} 171831$ & gi|167466275|ref|NM_152542.3 Homo sapiens protein phosphatase 1K (PP2C domain containing) (PPM1K), mRNA & 1.76709 \\
\hline DK582810 & gi|90652856|ref|NM_032818.2 Homo sapiens chromosome 9 open reading frame 100 (C9orf100), mRNA & 1.765 \\
\hline AB170534 & gi|108773786|ref|NM_000321.2 Homo sapiens retinoblastoma 1 (RB1), mRNA & 1.76182 \\
\hline AB171096 & $\begin{array}{r}\text { gi|110347436|ref|NM_001042545.1 Homo sapiens latent transform } \\
\text { transcript variant } 3, \mathrm{n}\end{array}$ & 1.75594 \\
\hline $\mathrm{AB} 168611$ & gi|21071068|ref|NM_004865.2 Homo sapiens TBP-like 1 (T & 1.74839 \\
\hline CJ492188 & gi|30181234|ref|NM_003447.2 Homo sapiens zinc finger protein 165 (ZNI & 1.74573 \\
\hline $\mathrm{AB} 171700$ & gi|115527063|ref|NM_004859.3 Homo sapiens clathrin, heavy chain (Hc) (CLTC), mRNA & 1.74566 \\
\hline $\mathrm{AB} 171366$ & gi|22748942|ref|NM_152445.1 Homo sapiens family with sequence similarity 161, member B (FAM161B), mRNA & 1.74405 \\
\hline AB168566 & gi|148664196|ref|NM_017950.2 Homo sapiens coiled-coil domain-containing 40 (CCDC40), mRNA & 1.74135 \\
\hline $\mathrm{AB} 171657$ & gi|221316692|ref|NM_198449.2 Homo sapiens embigin homolog (mouse) (EMB), mRNA & 1.73933 \\
\hline AB056808 & gi|71772767|ref|NM_152826.2 Homo sapiens-sorting nexin 1 (SNX1), transcript variant 3, mRNA & 1.73686 \\
\hline $\mathrm{AB} 168849$ & $\begin{array}{c}\text { gi|156766042|ref|NM_001103146.1 Homo sapiens GRB10-interacting GYF protein } 2 \text { (GIGYF2), } \\
\text { transcript variant 3, mRNA }\end{array}$ & 1.73412 \\
\hline $\mathrm{AB} 172848$ & $\begin{array}{l}\text { gi|95113665|ref|NM_018157.2 Homo sapiens resistance to inhibitors of cholinesterase } 8 \text { homolog B (C. ele } \\
\text { (RIC8B), mRNA }\end{array}$ & 1.72749 \\
\hline AB048894 & gi|148727250|ref|NM_007137.2 Homo sapiens zinc finger protein 81 (ZNF81), mRNA & 1.71845 \\
\hline DW524469 & gi|239753181|ref|XM_002345525.1 PREDICTED: Homo sapiens similar to hCG2041348 (LOC100293610), mRNA & 1.71582 \\
\hline $\mathrm{AB} 173566$ & gi|89242130|ref|NM_014305.2 Homo sapiens TDP-glucose 4,6-dehydratase (TGDS), mRNA & 1.71406 \\
\hline DC634783 & gi|116812576|ref|NM_016019.2 Homo sapiens LUC7-like 2 (S. cerevisiae) (LUC7L2), mRNA & 1.71163 \\
\hline $\mathrm{AB} 168438$ & gi|64276485|ref|NM_005869.2 Homo sapiens serologically defined colon cancer antigen 10 (SDCCAG10), mRNA & 1.71108 \\
\hline $\mathrm{AB} 174725$ & gi|169194555|ref|XR_040716.1 PREDICTED: Homo sapiens hypothetical LOC439950 (LOC439950), miscRNA & 1.70933 \\
\hline $\mathrm{AB} 170786$ & gi|208879448|ref|NM_006265.2 Homo sapiens RAD21 homolog (S. pombe) (RAD21), mRNA & 1.70551 \\
\hline CJ431422 & $\begin{array}{l}\text { gi|117938253|ref|NM_001077441.1 Homo sapiens BCL2-associated transcription factor 1 (BCLAF1), } \\
\text { transcript variant 3, mRNA }\end{array}$ & 1.70446 \\
\hline AB048954 & gi|148596971|ref|NM_014951.2 Homo sapiens zinc finger protein 365 (ZNF365), transcript variant A, mRNA & 1.70334 \\
\hline $\mathrm{AB} 173447$ & gi|40288292|ref|NM_000361.2 Homo sapiens thrombomodulin (THBD), mRNA & 1.70293 \\
\hline AB173287 & gi|242117988|ref|NM_014702.4 Homo sapiens KIAA0408 (KIAA0408), mRNA & 1.70162 \\
\hline CJ489820 & $\begin{array}{c}\text { gi|218505834|ref|NM_001142782.1 Homo sapiens membrane-associated guanylate kinase, WW, and PDZ } \\
\text { domain-containing } 3 \text { (MAGI3), transcript variant 1, mRNA }\end{array}$ & 1.68381 \\
\hline $\mathrm{AB} 173372$ & $\begin{array}{c}\text { gi|78190481|ref|NM_025221.5 Homo sapiens Kv channel-interacting protein } 4 \text { (KCNIP4), transcript } \\
\text { variant 1, mRNA }\end{array}$ & 1.68058 \\
\hline $\mathrm{AB} 172865$ & $\begin{array}{c}\text { gi|31795545|ref|NM_012450.2 Homo sapiens solute carrier family } 13 \text { (sodium/sulfate symporters), } \\
\text { member } 4 \text { (SLC13A4), mRNA }\end{array}$ & 1.67878 \\
\hline
\end{tabular}


TABle 1: Continued.

\begin{tabular}{|c|c|c|}
\hline $\begin{array}{l}\text { Accession } \\
\text { number }\end{array}$ & Human RefSeq description & $\begin{array}{c}\text { Fold } \\
\text { change }\end{array}$ \\
\hline AB168329 & $\begin{array}{c}\text { gi|223468562|ref|NM_005628.2 Homo sapiens solute carrier family } 1 \text { (neutral amino acid transporter), } \\
\text { member } 5 \text { (SLC1A5), transcript variant 1, mRNA }\end{array}$ & 1.67642 \\
\hline $\mathrm{AB} 171546$ & $\begin{array}{c}\text { gi|55956903|ref|NM_005922.2 Homo sapiens mitogen-activated protein kinase kinase kinase } 4 \text { (MAP3K4), } \\
\text { transcript variant 1, mRNA }\end{array}$ & 1.67151 \\
\hline AB063093 & gi|194248055|ref|NM_002045.3 Homo sapiens growth-associated protein 43 (GAP43), transcript variant 2, mRNA & 1.66805 \\
\hline AB220449 & $\begin{array}{c}\text { gi|23510394|ref|NM_138966.2 Homo sapiens neuropilin- (NRP-) and tolloid- (TLL-) like } 1 \text { (NETO1), } \\
\text { transcript variant 3, mRNA }\end{array}$ & 1.66789 \\
\hline AB169208 & gi|22547155|ref|NM_002018.2 Homo sapiens flightless I homolog (Drosophila) (FLII), mRNA & 1.66361 \\
\hline $\mathrm{AB} 168324$ & gi|116014337|ref|NM_030981.2 Homo sapiens RAB1B, member RAS oncogene family (RAB1B), mRNA & 1.66298 \\
\hline AB169835 & gi|50726964|ref|NM_013392.2 Homo sapiens nuclear receptor-binding protein 1 (NRBP1), mRNA & 1.65785 \\
\hline $\mathrm{AB} 173501$ & gi|195539333|ref|NM_018176.3 Homo sapiens leucine-rich repeat LGI family, member 2 (LGI2), mRNA & 1.6574 \\
\hline DC630946 & $\begin{array}{c}\text { gi|183227689|ref|NM_002049.3 Homo sapiens GATA-binding protein } 1 \text { (globin transcription factor 1) } \\
\text { (GATA1), mRNA }\end{array}$ & 1.65657 \\
\hline AB063075 & gi|239743824|ref|XM_001128647.3 PREDICTED: Homo sapiens hypothetical LOC728701 (LOC728701), mRNA & 1.65564 \\
\hline $\mathrm{AB} 169782$ & $\begin{array}{c}\text { gi|38261964|ref|NM_198399.1 Homo sapiens cyclic AMP-regulated phosphoprotein, } 21 \text { kD (ARPP-21), } \\
\text { transcript variant 2, mRNA }\end{array}$ & 1.65067 \\
\hline CJ477467 & gi|133778911|ref|NM_003309.2 Homo sapiens TSPY-like 1 (TSPYL1), mRNA & 1.65062 \\
\hline BB900725 & gi|31542685|ref|NM_025125.2 Homo sapiens chromosome 10 open reading frame 57 (C10orf57), mRNA & 1.64409 \\
\hline AB220555 & $\begin{array}{c}\text { gi|208973250|ref|NM_003702.3 Homo sapiens regulator of G-protein signaling } 20 \text { (RGS20), transcript } \\
\text { variant 2, mRNA }\end{array}$ & 1.64376 \\
\hline $\mathrm{AB} 171804$ & gi|66932910|ref|NM_014676.2 Homo sapiens pumilio homolog 1 (Drosophila) (PUM1), transcript variant 2, mRNA & 1.63724 \\
\hline DC625559 & gi|4557320|ref|NM_000039.1 Homo sapiens apolipoprotein A-I (APOA1), mRNA & 1.631 \\
\hline $\mathrm{AB} 172266$ & gi|170932491|ref|NM_030770.2 Homo sapiens transmembrane protease, serine 5 (TMPRSS5), mRNA & 1.62995 \\
\hline AB173763 & $\begin{array}{c}\text { gi|62953115|ref|NM_001017523.1 Homo sapiens BTB (POZ) domain-containing } 11 \text { (BTBD11), transcript } \\
\text { variant b, mRNA }\end{array}$ & 1.62499 \\
\hline AB172974 & gi|111161293|ref|NM_005746.2 Homo sapiens nicotinamide phosphoribosyltransferase (NAMPT), mRNA & 1.62078 \\
\hline AB179155 & gi|187608347|ref|NM_145046.3 Homo sapiens calreticulin 3 (CALR3), mRNA & 1.6111 \\
\hline AB169148 & gi|153792481|ref|NM_033048.4 Homo sapiens CPX chromosome region, candidate 1 (CPXCR1), mRNA & 1.60985 \\
\hline $\mathrm{AB} 171264$ & gi|130977817|ref|NM_024549.4 Homo sapiens tectonic family member 1 (TCTN1), transcript variant 3, mRNA & 1.60704 \\
\hline AB172446 & $\begin{array}{c}\text { gi|193083128|ref|NM_001128920.1 Homo sapiens MAP/microtubule affinity-regulating kinase } 3 \text { (MARK3), } \\
\text { transcript variant 4, mRNA }\end{array}$ & 1.60471 \\
\hline DC852298 & $\begin{array}{c}\text { gi|195972796|ref|NM_001130917.1 Homo sapiens leukocyte immunoglobulin-like receptor, subfamily A } \\
\text { (with TM domain), member } 2 \text { (LILRA2), transcript variant 1, mRNA }\end{array}$ & 1.59852 \\
\hline AB046637 & $\begin{array}{c}\text { gi|209571546|ref|NM_018095.4 Homo sapiens Kelch repeat and BTB (POZ) domain-containing } 4 \text { (KBTBD4), } \\
\text { transcript variant 1, mRNA }\end{array}$ & 1.58107 \\
\hline CJ445723 & gi|90903230|ref|NM_002111.6 Homo sapiens huntingtin (HTT), mRNA & 1.57689 \\
\hline DC630899 & $\begin{array}{l}\text { gi|83641894|ref|NM_031157.2 Homo sapiens heterogeneous nuclear ribonucleoprotein A1 (HNRNPA1), } \\
\text { transcript variant 2, mRNA }\end{array}$ & 1.5766 \\
\hline $\mathrm{AB} 168476$ & gi|219555742|ref|NM_015335.3 Homo sapiens mediator complex subunit 13-like (MED13L), mRNA & 1.57355 \\
\hline DC642541 & $\begin{array}{c}\text { gi|20357546|ref|NM_004231.2 Homo sapiens ATPase, H+ transporting, lysosomal } 14 \text { kDa, V1 subunit F } \\
\text { (ATP6V1F), mRNA }\end{array}$ & 1.57215 \\
\hline $\mathrm{AB} 170370$ & $\begin{array}{c}\text { gi|224177554|ref|NM_002340.5 Homo sapiens lanosterol synthase (2,3-oxidosqualene-lanosterol cyclase) (LSS), } \\
\text { transcript variant 1, mRNA }\end{array}$ & 1.57006 \\
\hline DC636538 & gi|71164876|ref|NM_001014.3 Homo sapiens ribosomal protein S10 (RPS10), mRNA & 1.56413 \\
\hline DC648258 & gi|4557818|ref|NM_000277.1 Homo sapiens phenylalanine hydroxylase (PAH), mRNA & 1.56396 \\
\hline AB168688 & $\begin{array}{l}\text { gi|75709218|ref|NM_001324.2 Homo sapiens cleavage stimulation factor, } 3^{\prime} \text { pre-RNA, subunit 1, } 50 \mathrm{kDa} \\
\text { (CSTF1), transcript variant 2, mRNA }\end{array}$ & 1.56349 \\
\hline CJ486539 & gi|194018543|ref|NM_031451.4 Homo sapiens testis expressed 101 (TEX101), transcript variant 1, mRNA & 1.55338 \\
\hline $\mathrm{AB} 173591$ & gi|56699472|ref|NM_006298.2 Homo sapiens zinc finger protein 192 (ZNF192), mRNA & 1.54892 \\
\hline $\mathrm{AB} 168460$ & gi|56090619|ref|NM_001007531.1 Homo sapiens NFKB-activating protein-like (NKAPL), mRNA & 1.54807 \\
\hline
\end{tabular}


TABle 1: Continued.

\begin{tabular}{|c|c|c|}
\hline $\begin{array}{l}\text { Accession } \\
\text { number }\end{array}$ & Human RefSeq description & $\begin{array}{c}\text { Fold } \\
\text { change }\end{array}$ \\
\hline AB046102 & $\begin{array}{c}\text { gi|215272394|ref|NM_001080475.2 Homo sapiens pleckstrin homology domain containing, family M, } \\
\text { member } 3 \text { (PLEKHM3), mRNA }\end{array}$ & 1.53926 \\
\hline AB097526 & gi|46409303|ref|NM_207332.1 Homo sapiens glutamate-rich 1 (ERICH1), mRNA & 1.53642 \\
\hline AB052134 & gi|227430412|ref|NM_024827.3 Homo sapiens histone deacetylase 11 (HDAC11), transcript variant 1, mRNA & 1.53543 \\
\hline AB170181 & $\begin{array}{c}\text { gi|21265090|ref|NM_007208.2 Homo sapiens mitochondrial ribosomal protein L3 (MRPL3), nuclear gene } \\
\text { encoding mitochondrial protein, mRNA }\end{array}$ & 1.52903 \\
\hline AB171241 & gi|116235443|ref|NM_138421.2 Homo sapiens serum amyloid A-like 1 (SAAL1), mRNA & 1.52713 \\
\hline AB171237 & gi|48675815|ref|NM_015723.2 Homo sapiens patatin-like phospholipase domain-containing 8 (PNPLA8), mRNA & 1.52587 \\
\hline DC625517 & $\begin{array}{c}\text { gi|47578120|ref|NM_177947.2 Homo sapiens armadillo repeat containing, X-linked } 3 \text { (ARMCX3), transcript } \\
\text { variant 2, mRNA }\end{array}$ & 1.52547 \\
\hline AB168964 & $\begin{array}{l}\text { gi|87159814|ref|NM_001696.3 Homo sapiens ATPase, H+ transporting, lysosomal } 31 \mathrm{kDa} \text {, V1 subunit E1 } \\
\text { (ATP6V1E1), transcript variant 1, mRNA }\end{array}$ & 1.52424 \\
\hline DC631115 & $\begin{array}{l}\text { gi|239752151|ref|XM_002348112.1 PREDICTED: Homo sapiens similar to immunoglobulin lambda locus } \\
\text { (LOC100290481), mRNA }\end{array}$ & 1.51711 \\
\hline DC640134 & $\begin{array}{l}\text { gi|208609986|ref|NM_014655.2 Homo sapiens solute carrier family 25, member } 44 \text { (SLC25A44), transcript } \\
\text { variant 1, mRNA }\end{array}$ & 1.51672 \\
\hline AB173691 & gi|94536855|ref|NM_013301.2 Homo sapiens coiled-coil domain-containing 106 (CCDC106), mRNA & 1.50477 \\
\hline AB168370 & gi|154744869|ref|NM_022752.5 Homo sapiens zinc finger protein 574 (ZNF574), mRNA & 1.50452 \\
\hline
\end{tabular}

spin. FITC fluorescence of TM cells was analyzed using a Cell Sorter SH800 (Sony Biotechnology, Tokyo, Japan).

2.5. Cell Viability Assay. The effects of Y-27632 on TM cell viability were evaluated using the WST-8 assay (Cell Counting Kit-8, Dojindo Laboratories, Kumamoto, Japan). Cells were seeded on 96-well plates $\left(1 \times 10^{4}\right.$ cells/well $)$ and incubated at $37^{\circ} \mathrm{C}$ under $5 \% \mathrm{CO}_{2}$ overnight. After pretreatment with Y-27632 for $30 \mathrm{~min}$, cells were stimulated with $\mathrm{H}_{2} \mathrm{O}_{2}$ or menadione for $24 \mathrm{~h}$. CCK- 8 reagents were added into each well and incubated for $2 \mathrm{~h}$ at $37^{\circ} \mathrm{C}$. Absorbance at $450 \mathrm{~nm}$ was determined using a microplate reader (Multiskan FC, Thermo Fisher Scientific). Cell viability was expressed as a percentage of control (vehicle-treated) cells.

2.6. Direct Antioxidant Activity of Y-27632. Direct antioxidant activity was assessed by 2-methyl-6-p-methoxyphenylethynylimidazopyrazinone (AB-2950 MPEC; ATTO, Tokyo, Japan), a superoxide-sensitive luminescent reagent, and reagents for xanthine-oxidase-induced superoxide production (AB-2970 CLETA-S, ATTO) following the manufacturer's protocol. Briefly, $10 \mu \mathrm{L}$ of $300 \mu \mathrm{M}$ MPEC/ethanol and $80 \mu \mathrm{L}$ of $1.25 \mathrm{unit} / \mathrm{mL}$ xanthine oxidase/HEPES were mixed. Then, $10 \mu \mathrm{L}$ of $25 \mu \mathrm{M}$ Y-27632 or $20 \mathrm{mM} \mathrm{n}$-acetyl cysteine (positive control) was added into each well of a 96-well plate. Subsequently, $90 \mu \mathrm{L}$ of the mixture of MPEC and xanthine oxidase and $200 \mu \mathrm{L}$ of xanthine were added to each well. The luminescent signal was measured for $10 \mathrm{~s}$ by a luminometer (AB-2270 Octa; ATTO).

2.7. Statistical Analysis. Data are presented as means \pm standard error. Statistical comparisons of multiple groups were performed using the Tukey-Kramer HSD test and Dunnett's test, and those of two groups were performed using Wilcoxon rank sum test and Wilcoxon signed rank test. Differences were considered statistically significant at $P<0.05$.

\section{Results}

3.1. Microarray Expression Profile in Y-27632-Treated TM Cells. Among the 12,613 genes analyzed by microarray, the affected genes are listed in Tables 1 and 2; 444 genes were upregulated, and 56 were downregulated. Significantly upregulated and downregulated gene categories based on gene ontology analysis in Y-27632 treated TM cells are listed in Tables 3 and 4. Gene ontology analysis revealed that the upregulated genes were related to various cellular functions including antioxidant activity $(P=0.014)$, and downregulated genes were related to integrin complexes $(P=0.039)$, and calcium ion transport into the cytosol $(P=0.008)$. In the category of antioxidant activity, upregulated genes were homologous to human gene coding catalase $(P=0.046)$, thioredoxin domain-containing 2 (also known as spermatozoa; $P=0.032)$, nucleoredoxin $(P=0.017)$, albumin (probe $1, P=0.002$; probe $2, P=0.021$ ), and glutathione transferase zeta $1(P=0.004)$. Upregulation of the mRNA of catalase, an extensively investigated antioxidant, was confirmed by real-time RT-PCR and found to be 1.5 times higher in TM cells treated with Y-27632 compared to the control TM cells $(P=0.032$; Figure $1(\mathrm{a}))$. In contrast, four other genes involved in antioxidant activity were not significantly affected after treatment with Y-27632 (data not shown).

3.2. Effects of Y-27632 on the Production of Reactive Oxygen Species in TM Cells. To assess the effects of Y-27632 on the production of ROS in TM cells, we utilized a fluorogenic probe that exhibits bright fluorescence upon oxidation by ROS. In the absence of an oxidative reagent, the fluorescence 
TABLE 2: Genes that are downregulated in TM cells.

\begin{tabular}{|c|c|c|}
\hline $\begin{array}{l}\text { Accession } \\
\text { number }\end{array}$ & Human RefSeq description & $\begin{array}{c}\text { Fold } \\
\text { change }\end{array}$ \\
\hline DC624859 & gi|215982788|ref|NM_000477.5 Homo sapiens albumin (ALB), mRNA & 0.11952 \\
\hline $\mathrm{AB} 171761$ & gi|148271103|ref|NM_173495.2 Homo sapiens patched domain-containing 1 (PTCHD1), mRNA & 0.13543 \\
\hline AB047615 & $\begin{array}{l}\text { gi|70780382|ref|NM_004285.3 Homo sapiens hexose-6-phosphate dehydrogenase (glucose 1-dehydrogenase) } \\
\text { (H6PD), mRNA }\end{array}$ & 0.17649 \\
\hline DC621007 & gi|38016905|ref|NR_001578.1 Homo sapiens L-threonine dehydrogenase (TDH), noncoding RNA & 0.20061 \\
\hline CJ443677 & gi|38327038|ref|NM_002154.3 Homo sapiens heat shock 70 kDa protein 4 (HSPA4), mRNA & 0.2086 \\
\hline DC622138 & gi|145386530|ref|NM_001084392.1 Homo sapiens D-dopachrome tautomerase (DDT), transcript variant 2, mRNA & 0.21345 \\
\hline BB891761 & $\begin{array}{l}\text { gi|33519462|ref|NM_004544.2 Homo sapiens NADH dehydrogenase (ubiquinone) } 1 \text { alpha subcomplex, 10, } \\
\text { 42 kDa (NDUFA10), nuclear gene encoding mitochondrial protein, mRNA }\end{array}$ & 0.23147 \\
\hline CJ444181 & $\begin{array}{r}\text { gi|226437566|ref|NM_001018060.2 Homo sapiens apoptosis-inducir } \\
\text { nuclear gene encoding mitochondrial protein, }\end{array}$ & 0.24045 \\
\hline $\mathrm{AB} 171890$ & gi|118572602|ref|NM_001079514.1 Homo sapiens ubinuclein 1 (UBN1), transcript variant 2, mRNA & 0.24676 \\
\hline $\mathrm{AB} 174511$ & gi|153792041|ref|NM_020823.1 Homo sapiens transmembrane protein 181 (TMEM181), mRNA & 0.29472 \\
\hline AB168319 & gi|116256484|ref|NM_006781.3 Homo sapiens chromosome 6 open reading frame 10 (C6orf10), mRNA & 0.3105 \\
\hline DW526909 & gi|20302159|ref|NM_005999.2 Homo sapiens translin-associated factor X (TSNAX), mRNA & 0.32949 \\
\hline AB173471 & $\begin{array}{r}\text { gi|154354995|ref|NM_002222.4 Homo sapiens inositol 1,4,5 } \\
\text { transcript variant } 2, \mathrm{mR}\end{array}$ & 0.33892 \\
\hline BB898986 & gi|167003944|ref|NM_000204.3 Homo & \\
\hline DK578390 & gi|56788350|ref|NM_001008695.1 Homo sapiens THAP & 0.35502 \\
\hline CJ444326 & $\begin{array}{r}\text { gi|209413724|ref|NM_003692.3 Homo sapiens transr } \\
\text { domains } 1 \text { (T }\end{array}$ & 0.3719 \\
\hline B048874 & gi|239753181|ref|XM_002345525.1 PREDICTED: Homo s & 0.38524 \\
\hline C635743 & gi|239750740|ref|XM_002347480.1 PREDICTED: Homo & 0.3882 \\
\hline CJ442045 & gi|96975096|ref|NM_016577.3 Homo sapiens RAB6B, men & 0.39034 \\
\hline BB897881 & gi|31542685|ref|NM_025125.2 Homo sapiens chromosome 10 open reading frame 57 (C10o & 0.39308 \\
\hline $\mathrm{AB} 168422$ & gi|194306536|ref|NM_144594.2 Homo sapiens gametocyte-specific factor 1 (GTSF1), & 0.43061 \\
\hline DW524779 & $\begin{array}{c}\text { gi|226342870|ref|NR_027449.1 Homo sapiens TBC1 domain family, member } 15 \text { (TBC1D15), transcript va } \\
\text { transcribed RNA }\end{array}$ & 0.44075 \\
\hline C630545 & gi|39812105|ref|NM_198941.1 Homo sapiens serine incorporator 3 (SERINC3), transcript variant 2, mRNA & 0.44508 \\
\hline AB172901 & gi|42544225|ref|NM_020857.2 Homo sapiens vacuolar protein sorting 18 homolog (S. cerevisiae) (VPS18), mRNA & 0.44872 \\
\hline DW528888 & $\begin{array}{c}\text { gi|40068463|ref|NM_020732.2 Homo sapiens AT-rich interactive domain 1B (SWI1-like) (ARID1B), transc } \\
\text { variant 2, mRNA }\end{array}$ & 0.4498 \\
\hline DC636880 & gi|17738314|ref|NM_006835 & 0.45196 \\
\hline AB220379 & gi|185134767|ref|NM_002524.3 Homo sapiens neuroblastoma RAS viral (v-ras) oncogene homolog (NRAS), mRNA & 6121 \\
\hline AB055316 & gi|226437631|ref|NM_001004339.2 Homo sapiens zyg-11 homolog A (C. elegans) (ZYG11A), mRNA & 0.46399 \\
\hline DC641070 & gi|58331227|ref|NM_005223.3 Homo sapiens deoxyribonuclease I (DNASE1), mRNA & 6575 \\
\hline AB056428 & gi|49574533|ref|NM_032782.3 Homo sapiens hepatitis A virus cellular receptor 2 (HAVCR2), mRNA & 0.46705 \\
\hline AB168577 & gi|146260272|ref|NM_001085451.1 Homo sapiens leukemia NUP98 fusion partner 1 (LNP1), mRNA & 0.46891 \\
\hline AB048999 & $\begin{array}{l}\text { gi|225735571|ref|NR_027416.1 Homo sapiens nuclear factor erythroid-derived 2-like } 3 \text { pseudogene } \\
\text { (LOC100272146), noncoding RNA }\end{array}$ & 0.4782 \\
\hline AB174085 & gi|142360382|ref|NM_176815.3 Homo sapiens dihydrofolate reductase-like 1 (DHFRL1), mRNA & 0.4904 \\
\hline DC642335 & $\begin{array}{c}\text { gi|148491081|ref|NM_001343.2 Homo sapiens-disabled homolog 2, mitogen-responsive phosphoprotein } \\
\text { (Drosophila) (DAB2), mRNA }\end{array}$ & 0.4971 \\
\hline $\mathrm{AB} 173771$ & gi|38176290|ref|NM_001233.3 Homo sapiens caveolin 2 (CAV2), transcript variant 1, mRNA & 0.49863 \\
\hline DW523198 & gi|145312264|ref|NM_033266.3 Homo sapiens endoplasmic reticulum to nucleus signaling 2 (ERN2), mRNA & 0.50158 \\
\hline AF492282 & $\begin{array}{c}\text { gi|52630343|ref|NM_021983.4 Homo sapiens major histocompatibility complex, class II, DR beta } 4 \\
\text { (HLA-DRB4), mRNA }\end{array}$ & 0.50533 \\
\hline AB047937 & gi|194097480|ref|NM_020412.4 Homo sapiens chromatin-modifying protein 1B (CHMP1B), mRNA & 0.51198 \\
\hline AB179165 & gi|118136291|ref|NM_006465.2 Homo sapiens AT-rich interactive domain 3B (bright-like) (ARID3B), mRNA & 0.51349 \\
\hline AJ585530 & gi|75709168|ref|NM_002260.3 Homo sapiens killer cell lectin-like receptor subfamily C, member 2 (KLRC2), mRNA & 0.52335 \\
\hline
\end{tabular}


TABLE 2: Continued.

\begin{tabular}{|c|c|c|}
\hline $\begin{array}{l}\text { Accession } \\
\text { number }\end{array}$ & Human RefSeq description & $\begin{array}{l}\text { Fold } \\
\text { change }\end{array}$ \\
\hline $\mathrm{AB} 170944$ & gi|40255250|ref|NM_144635.3 Homo sapiens family with sequence similarity 131, member A (FAM131A), mRNA & 0.55244 \\
\hline AB171281 & $\begin{array}{c}\text { gi|154759258|ref|NM_003127.2 Homo sapiens spectrin, alpha, nonerythrocytic } 1 \text { (alpha-fodrin) (SPTAN1), } \\
\text { transcript variant 2, mRNA }\end{array}$ & 0.555 \\
\hline $\mathrm{AB} 172429$ & $\begin{array}{c}\text { gi|224994204|ref|NM_001145853.1 Homo sapiens Wolfram syndrome } 1 \text { (wolframin) (WFS1), transcript } \\
\text { variant 2, mRNA }\end{array}$ & 0.57755 \\
\hline $\mathrm{AB} 171421$ & gi|110611175|ref|NM_000843.3 Homo sapiens glutamate receptor, metabotropic 6 (GRM6), mRNA & 0.58226 \\
\hline AB096987 & $\begin{array}{c}\text { gi|239750853|ref|XR_079356.1 PREDICTED: Homo sapiens hypothetical protein LOC100130855 } \\
\text { (LOC100130855), miscRNA }\end{array}$ & 0.59997 \\
\hline AB049000 & gi|209977116|ref|NM_080872.2 Homo sapiens unc-5 homolog D (C. elegans) (UNC5D), mRNA & 0.60066 \\
\hline BB893759 & gi|194018407|ref|NM_178148.2 Homo sapiens solute carrier family 35, member B2 (SLC35B2), mRNA & 0.61849 \\
\hline AB056791 & gi|162951883|ref|NM_014925.3 Homo sapiens R3H domain-containing 2 (R3HDM2), mRNA & 0.64652 \\
\hline AB070176 & gi|221307501|ref|NM_001143976.1 Homo sapiens WEE1 homolog (S. pombe) (WEE1), transcript variant 2, mRNA & 0.64704 \\
\hline AB048962 & $\begin{array}{c}\text { gi|154146186|ref|NM_152634.2 Homo sapiens transcription elongation factor A (SII) N-terminal and central } \\
\text { domain containing (TCEANC), mRNA }\end{array}$ & 0.65673 \\
\hline DK578501 & $\begin{array}{c}\text { gi|24797073|ref|NM_033554.2 Homo sapiens major histocompatibility complex, class II, DP alpha } 1 \\
\text { (HLA-DPA1), mRNA }\end{array}$ & 0.65963 \\
\hline
\end{tabular}

intensity was not significantly different in TM cells treated with Y-27632 compared to control $(3673.2 \pm 452.3$ versus $5104.5 \pm 735.0$; Figure 1(b)). In the presence of $100 \mu \mathrm{M}$ menadione, the fluorescence intensity was significantly elevated $(16097.7 \pm 1133.0 ; \quad P<0.0001)$; this elevation was partly suppressed by treatment with Y-27632 (11443.6 \pm 1332.2 ; $P=0.0182$ ), suggesting that $\mathrm{Y}-27632$ reduces ROS production in TM cells under oxidative stress.

3.3. Effects of Y-27632 on the Viability of TM Cells under Oxidative Stress. Finally, we investigated the effects of Y-27632 on the viability of TM cells under oxidative stress. As shown in Figure 2(a), menadione reduced TM cell viability in a dose-dependent manner. At a lower dose of menadione, Y-27632-stimulated TM cells regained significant viability against menadione treatment compared to control cells $(P=0.0238)$. In contrast, the effects of Y-27632 on cell viability were not significant at a higher dose of menadione.

3.4. Direct Antioxidant Activity of Y-27632. To confirm the extracellular antioxidant activity of Y-27632, we assessed xanthine oxidase-induced superoxide production using a luminescent reagent. As shown in Figure 2(b), there was no significant difference in ROS production between the control and Y-27632 treatment. Thus, Y-27632 does not appear to affect extracellular oxidants.

\section{Discussion}

In the present study, we have identified the antioxidative effect of Y-27632 in TM cells by microarray analysis, an exhaustive investigation of gene expression, and shown that Y-27632 partially suppresses ROS production and cell death induced by menadione. To the best of our knowledge, this is the first report to show the antioxidant effect of ROCK inhibitor on TM cells. Previously, we presented depolymerization of F-actin before morphometric recovery from oxidative stress in TM cells [24], suggesting a correlation between oxidative stress and regulation of the actin cytoskeleton in TM cells. In other tissues, rho-kinase was identified as a mediator of various diseases associated with inflammation and oxidative stress, and inhibition of rhokinase has been drawing attention as a promising therapeutic strategy. For instance, activation of the rho/rho-kinase pathway is related to the pathophysiology of chronic renal injury, and long-term fasudil treatment has renoprotective effects in this malignant hypertension model. The mechanism of the renoprotective effect of fasudil, a nonspecific ROCK inhibitor, was suggested to involve a combination of factors, including inhibition of the TGF- $\beta$-collagen cascade, control of inflammation, reduction of oxidative stress, and upregulation of eNOS [18]. Clinical studies with fasudil have suggested that it may be useful for the treatment of a wide range of cardiovascular diseases [19]. Importantly, rhokinase inhibitors block ROS production by suppressing CyPA secretion from vascular smooth muscle cells [25], suggesting the beneficial effect of rho-kinase inhibitors against cardiovascular diseases.

Recently, Yamamoto and colleagues demonstrated the neuroprotective effect of the ROCK inhibitor K-115, a novel IOP-lowering drug, using the mouse optic crush model [20]. They showed the effect was at least partially dependent on suppression of ROS production via inhibition of Nox1 expression in retinal ganglion cells. We also showed that ROCK inhibitors' antioxidant effects are indirect using monkey TM cells. However, in the present study using microarray analysis, Nox family genes were not identified as affected, but catalase was upregulated after treatment with Y-27632. This disagreement might be caused by differences in species and/or tissues. Thus, the precise molecular mechanisms of the antioxidative effect of ROCK inhibitors have not been clarified completely. On the other hand, a recent study 
TABLE 3: Gene ontology of upregulated genes in Y-27632-treated TM cells.

\begin{tabular}{|c|c|c|c|c|}
\hline Ontology & Term & Changed genes & Total genes & $P$ value \\
\hline Cellular component & Cell projection & $31(1)$ & $331(38)$ & 0.0000306 \\
\hline Cellular component & Neuron projection & $20(6)$ & $180(28)$ & 0.0000901 \\
\hline Cellular component & Cell projection part & $17(0)$ & $142(0)$ & 0.000135 \\
\hline Biological process & Regulation of neurotransmitter levels & $8(0)$ & $47(2)$ & 0.00148 \\
\hline Molecular function & Calcium channel regulator activity & $4(3)$ & $11(9)$ & 0.00225 \\
\hline Cellular component & Presynaptic membrane & $5(5)$ & $20(20)$ & 0.0024 \\
\hline Cellular component & Plasma membrane part & $45(0)$ & $706(2)$ & 0.00259 \\
\hline Biological process & Synaptic transmission & $16(10)$ & $170(66)$ & 0.00374 \\
\hline Molecular function & Channel regulator activity & $5(0)$ & $23(0)$ & 0.00421 \\
\hline Biological process & Cellular nitrogen compound biosynthetic process & $21(0)$ & $255(0)$ & 0.00443 \\
\hline Biological process & L-Glutamate import & $3(3)$ & $6(4)$ & 0.00456 \\
\hline Cellular component & Platelet alpha granule & $5(1)$ & $24(2)$ & 0.00472 \\
\hline Biological process & Transmission of nerve impulse & $17(0)$ & $189(3)$ & 0.00563 \\
\hline Molecular function & Anion:cation symporter activity & $4(0)$ & $15(0)$ & 0.00565 \\
\hline Molecular function & Sodium:dicarboxylate symporter activity & $3(3)$ & $7(7)$ & 0.00585 \\
\hline Biological process & L-Amino acid import & $3(0)$ & $7(0)$ & 0.00632 \\
\hline Biological process & Amino acid import & $3(0)$ & $7(0)$ & 0.00632 \\
\hline Cellular component & Axon part & $6(0)$ & $38(2)$ & 0.00665 \\
\hline Biological process & Regulation of mitotic cell cycle & $8(1)$ & $62(6)$ & 0.00683 \\
\hline Molecular function & Anion transmembrane transporter activity & $8(0)$ & $66(5)$ & 0.00813 \\
\hline Biological process & Cell-cell signaling & $21(4)$ & $268(74)$ & 0.00841 \\
\hline Molecular function & High-affinity glutamate transmembrane transporter activity & $2(2)$ & $2(2)$ & 0.00875 \\
\hline Cellular component & Cytoplasmic vesicle part & $9(0)$ & $83(2)$ & 0.00918 \\
\hline Biological process & Deoxyribonucleoside triphosphate biosynthetic process & $2(0)$ & $2(0)$ & 0.00923 \\
\hline Biological process & Response to calcium ion & $5(4)$ & $28(25)$ & 0.00969 \\
\hline Molecular function & Rho guanyl-nucleotide exchange factor activity & $5(5)$ & $29(29)$ & 0.00984 \\
\hline Biological process & Carboxylic acid transport & $9(0)$ & $81(1)$ & 0.01005 \\
\hline Molecular function & Phosphatidylinositol binding & $3(3)$ & $9(9)$ & 0.01012 \\
\hline Molecular function & Dicarboxylic acid transmembrane transporter activity & $3(0)$ & $9(0)$ & 0.01012 \\
\hline Biological process & Organic acid transport & $9(0)$ & $82(0)$ & 0.01077 \\
\hline Biological process & Glutamate metabolic process & $3(0)$ & $9(4)$ & 0.01091 \\
\hline Biological process & Dicarboxylic acid transport & $3(3)$ & $9(7)$ & 0.01091 \\
\hline Cellular component & Axoneme & $4(2)$ & $19(5)$ & 0.01104 \\
\hline Molecular function & Structural constituent of cytoskeleton & $5(5)$ & $31(31)$ & 0.01251 \\
\hline Cellular component & Cytoplasmic membrane-bounded vesicle lumen & $4(0)$ & $20(0)$ & 0.01285 \\
\hline Cellular component & Platelet alpha granule lumen & $4(4)$ & $20(20)$ & 0.01285 \\
\hline Cellular component & Dendritic spine & $4(4)$ & $20(20)$ & 0.01285 \\
\hline Cellular component & Neuron spine & $4(0)$ & $20(0)$ & 0.01285 \\
\hline Cellular component & Axon & $9(6)$ & $89(62)$ & 0.01361 \\
\hline Cellular component & Neurofilament & $2(2)$ & $3(3)$ & 0.01394 \\
\hline Molecular function & Antioxidant activity & $5(2)$ & $32(12)$ & 0.01401 \\
\hline Biological process & Rho protein signal transduction & $7(1)$ & $57(15)$ & 0.01403 \\
\hline Molecular function & Phenylalanine 4-monooxygenase activity & $2(2)$ & $3(3)$ & 0.0142 \\
\hline Biological process & ER to Golgi vesicle-mediated transport & $4(4)$ & $20(20)$ & 0.01457 \\
\hline Cellular component & Vesicle lumen & $4(0)$ & $21(1)$ & 0.01484 \\
\hline Biological process & D-Amino acid transport & $2(0)$ & $3(0)$ & 0.01497 \\
\hline Biological process & D-Aspartate import & $2(2)$ & $3(3)$ & 0.01497 \\
\hline Biological process & D-Aspartate transport & $2(0)$ & $3(0)$ & 0.01497 \\
\hline Biological process & Glutamate biosynthetic process & $2(2)$ & $3(3)$ & 0.01497 \\
\hline
\end{tabular}


TABle 3: Continued.

\begin{tabular}{|c|c|c|c|c|}
\hline Ontology & Term & Changed genes & Total genes & $P$ value \\
\hline Biological process & 2'-Deoxyribonucleotide biosynthetic process & $2(0)$ & $3(0)$ & 0.01497 \\
\hline Biological process & Fatty acid transport & $4(1)$ & $21(6)$ & 0.01681 \\
\hline Molecular function & Ras guanyl-nucleotide exchange factor activity & $5(0)$ & $34(3)$ & 0.01737 \\
\hline Biological process & Regulation of cell cycle process & $6(0)$ & $47(0)$ & 0.01912 \\
\hline Biological process & Nucleoside triphosphate biosynthetic process & $8(1)$ & $76(3)$ & 0.0195 \\
\hline Molecular function & Transporter activity & $34(8)$ & $556(147)$ & 0.01993 \\
\hline Molecular function & Monocarboxylic acid binding & $4(0)$ & $23(0)$ & 0.02002 \\
\hline Cellular component & Neurofilament cytoskeleton & $2(0)$ & $4(1)$ & 0.02037 \\
\hline Biological process & Pyrimidine nucleoside triphosphate biosynthetic process & $3(0)$ & $12(0)$ & 0.02063 \\
\hline Biological process & Pyrimidine nucleoside triphosphate metabolic process & $3(0)$ & $12(0)$ & 0.02063 \\
\hline Molecular function & $\begin{array}{c}\text { Oxidoreductase activity, acting on paired donors, with incorporation } \\
\text { or reduction of molecular oxygen, reduced pteridine as one donor, } \\
\text { and incorporation of one atom of oxygen }\end{array}$ & $2(1)$ & $4(2)$ & 0.02075 \\
\hline Molecular function & Thioredoxin-disulfide reductase activity & $2(2)$ & $4(4)$ & 0.02075 \\
\hline Biological process & Regulation of secretion & $8(0)$ & $77(0)$ & 0.0208 \\
\hline Biological process & Neurotransmitter biosynthetic process & $2(2)$ & $4(4)$ & 0.02186 \\
\hline Biological process & Tetrahydrobiopterin metabolic process & $2(1)$ & $4(1)$ & 0.02186 \\
\hline Biological process & Deoxyribonucleoside triphosphate metabolic process & $2(0)$ & $4(1)$ & 0.02186 \\
\hline Cellular component & Clathrin coat & $4(1)$ & $24(1)$ & 0.02193 \\
\hline Biological process & Response to metal ion & $8(0)$ & $78(3)$ & 0.02216 \\
\hline Cellular component & Transport vesicle membrane & $3(0)$ & $13(0)$ & 0.02236 \\
\hline Biological process & Neurotransmitter metabolic process & $3(1)$ & $13(3)$ & 0.02465 \\
\hline Biological process & Long-chain fatty acid transport & $3(2)$ & $13(4)$ & 0.02465 \\
\hline Molecular function & Oxidoreductase activity, acting on sulfur group of donors & $4(0)$ & $25(0)$ & 0.02549 \\
\hline Molecular function & Organic acid:sodium symporter activity & $3(0)$ & $14(0)$ & 0.02707 \\
\hline Molecular function & Phosphoinositide binding & $6(2)$ & $53(30)$ & 0.02738 \\
\hline Molecular function & Carboxylic acid binding & $8(0)$ & $84(5)$ & 0.02754 \\
\hline Cellular component & MHC protein complex & $4(0)$ & $26(0)$ & 0.02764 \\
\hline Molecular function & DNA topoisomerase type I activity & $2(2)$ & $5(5)$ & 0.02831 \\
\hline Molecular function & Solute:sodium symporter activity & $4(0)$ & $26(0)$ & 0.02853 \\
\hline Cellular component & Synapse & $13(10)$ & $167(116)$ & 0.0289 \\
\hline Biological process & L-Amino acid transport & $3(0)$ & $14(1)$ & 0.02906 \\
\hline Biological process & Cilium morphogenesis & $3(1)$ & $14(3)$ & 0.02906 \\
\hline Cellular component & Secretory granule & $8(3)$ & $86(27)$ & 0.02929 \\
\hline Biological process & Positive regulation of myeloid leukocyte differentiation & $2(0)$ & $5(0)$ & 0.0298 \\
\hline Biological process & Glutamate catabolic process & $2(0)$ & $5(0)$ & 0.0298 \\
\hline Biological process & Sulfate transport & $2(2)$ & $5(5)$ & 0.0298 \\
\hline Biological process & Deoxyribonucleotide biosynthetic process & $2(0)$ & $5(2)$ & 0.0298 \\
\hline Cellular component & Endomembrane system & $26(1)$ & $416(14)$ & 0.03019 \\
\hline Cellular component & External side of plasma membrane & $6(5)$ & $55(49)$ & 0.03035 \\
\hline Cellular component & Plasma membrane & $64(48)$ & $1224(935)$ & 0.03044 \\
\hline Cellular component & Endocytic vesicle membrane & $3(3)$ & $15(12)$ & 0.03078 \\
\hline Cellular component & Clathrin coated vesicle membrane & $4(0)$ & $27(3)$ & 0.0308 \\
\hline Cellular component & Intrinsic to organelle membrane & $8(0)$ & $87(0)$ & 0.03095 \\
\hline Biological process & Regulation of rho protein signal transduction & $5(5)$ & $39(29)$ & 0.03101 \\
\hline Biological process & Neurotransmitter secretion & $4(3)$ & $26(11)$ & 0.03114 \\
\hline Cellular component & Synapse part & $10(0)$ & $117(2)$ & 0.03187 \\
\hline Biological process & Vitamin transport & $3(1)$ & $15(1)$ & 0.03386 \\
\hline Molecular function & Symporter activity & $6(6)$ & $57(44)$ & 0.03631 \\
\hline
\end{tabular}


TABle 3: Continued.

\begin{tabular}{|c|c|c|c|c|}
\hline Ontology & Term & Changed genes & Total genes & $P$ value \\
\hline Molecular function & Syntaxin-1 binding & $2(2)$ & $6(6)$ & 0.03678 \\
\hline Molecular function & Ion channel inhibitor activity & $2(1)$ & $6(2)$ & 0.03678 \\
\hline Biological process & Cell communication & $34(4)$ & $562(40)$ & 0.03717 \\
\hline Cellular component & Endocytic vesicle & $4(1)$ & $29(10)$ & 0.03772 \\
\hline Biological process & Regulation of mitosis & $4(2)$ & $28(7)$ & 0.03839 \\
\hline Biological process & Regulation of nuclear division & $4(0)$ & $28(0)$ & 0.03839 \\
\hline Biological process & Response to inorganic substance & $10(1)$ & $118(8)$ & 0.03843 \\
\hline Biological process & Phosphatidylcholine biosynthetic process & $2(2)$ & $6(5)$ & 0.03869 \\
\hline Biological process & Interleukin-1 beta secretion & $2(0)$ & $6(0)$ & 0.03869 \\
\hline Biological process & Interleukin-1 secretion & $2(0)$ & $6(0)$ & 0.03869 \\
\hline Biological process & Regulation of interleukin- 1 beta secretion & $2(0)$ & $6(0)$ & 0.03869 \\
\hline Biological process & Regulation of interleukin-1 secretion & $2(0)$ & $6(0)$ & 0.03869 \\
\hline Biological process & Cdc42 protein signal transduction & $2(1)$ & $6(3)$ & 0.03869 \\
\hline Biological process & L-Phenylalanine catabolic process & $2(2)$ & $6(6)$ & 0.03869 \\
\hline Biological process & L-Phenylalanine metabolic process & $2(0)$ & $6(0)$ & 0.03869 \\
\hline Biological process & Tyrosine metabolic process & $2(0)$ & $6(1)$ & 0.03869 \\
\hline Biological process & Multicellular organismal aging & $2(1)$ & $6(2)$ & 0.03869 \\
\hline Biological process & Aspartate transport & $2(0)$ & $6(3)$ & 0.03869 \\
\hline Biological process & $\begin{array}{c}\text { Negative regulation of transforming growth factor beta receptor } \\
\text { signaling pathway }\end{array}$ & $2(2)$ & $6(6)$ & 0.03869 \\
\hline Biological process & Neurofilament cytoskeleton organization & $2(1)$ & $6(5)$ & 0.03869 \\
\hline Molecular function & Chloride ion binding & $4(4)$ & $29(29)$ & 0.0389 \\
\hline Biological process & L-Glutamate transport & $3(0)$ & $16(7)$ & 0.03904 \\
\hline Biological process & Platelet activation & $3(2)$ & $16(11)$ & 0.03904 \\
\hline Biological process & Positive regulation of secretion & $5(0)$ & $42(1)$ & 0.03976 \\
\hline Molecular function & Substrate-specific transporter activity & $28(0)$ & $463(0)$ & 0.04034 \\
\hline Biological process & Nucleotide biosynthetic process & $12(0)$ & $151(7)$ & 0.04052 \\
\hline Cellular component & Microtubule basal body & $3(3)$ & $17(17)$ & 0.04062 \\
\hline Molecular function & Calcium-dependent protein binding & $3(3)$ & $17(17)$ & 0.04164 \\
\hline Biological process & Anion transport & $7(0)$ & $73(10)$ & 0.04185 \\
\hline Molecular function & Solute:cation symporter activity & $5(0)$ & $44(0)$ & 0.04201 \\
\hline Biological process & Purine nucleoside triphosphate biosynthetic process & $7(0)$ & $74(0)$ & 0.0443 \\
\hline Biological process & Acidic amino acid transport & $3(0)$ & $17(0)$ & 0.04461 \\
\hline Biological process & Pyrimidine nucleoside metabolic process & $3(0)$ & $17(1)$ & 0.04461 \\
\hline Biological process & Pyrimidine nucleotide biosynthetic process & $3(0)$ & $17(4)$ & 0.04461 \\
\hline Cellular component & MHC class II protein complex & $3(3)$ & $18(18)$ & 0.04607 \\
\hline Molecular function & $\begin{array}{l}\text { Oxidoreductase activity, acting on sulfur group of donors, } \\
\text { NAD, or NADP as acceptor }\end{array}$ & $2(0)$ & $7(2)$ & 0.04608 \\
\hline Molecular function & Fatty acid transporter activity & $2(1)$ & $7(3)$ & 0.04608 \\
\hline Molecular function & Channel inhibitor activity & $2(0)$ & $7(1)$ & 0.04608 \\
\hline Molecular function & DNA topoisomerase activity & $2(1)$ & $7(2)$ & 0.04608 \\
\hline Biological process & Regulation of cytokine production & $7(0)$ & $75(0)$ & 0.04684 \\
\hline Molecular function & NADP or NADPH binding & $3(3)$ & $18(17)$ & 0.04721 \\
\hline Biological process & Nucleobase, nucleoside, and Nucleotide biosynthetic process & $12(0)$ & $157(0)$ & 0.04725 \\
\hline Biological process & Nucleobase, nucleoside, nucleotide, and nucleic acid biosynthetic process & $12(0)$ & $157(0)$ & 0.04725 \\
\hline Biological process & G1 phase & $2(1)$ & $7(1)$ & 0.04845 \\
\hline Cellular component & Lamellipodium & $4(4)$ & $32(32)$ & 0.04965 \\
\hline Cellular component & Transport vesicle & $4(1)$ & $32(11)$ & 0.04965 \\
\hline
\end{tabular}


TABLE 4: Gene ontology of downregulated genes in Y-27632-treated TM cells.

\begin{tabular}{|c|c|c|c|c|}
\hline Ontology & Term & Changed genes & Total genes & $P$ value \\
\hline Molecular function & $\begin{array}{c}\text { Endonuclease activity, active with either ribo- or deoxyribonucleic } \\
\text { acids and producing } 5 \text {-phosphomonoesters }\end{array}$ & $2(0)$ & $13(0)$ & 0.00279 \\
\hline Biological process & $\begin{array}{c}\text { Antigen processing and presentation of peptide or polysaccharide } \\
\text { antigen via MHC class II }\end{array}$ & $2(2)$ & $16(14)$ & 0.00421 \\
\hline Cellular component & MHC class II protein complex & $2(2)$ & $18(18)$ & 0.00606 \\
\hline Molecular function & D-Dopachrome decarboxylase activity & $1(1)$ & $1(1)$ & 0.01065 \\
\hline Molecular function & Glucose 1-dehydrogenase activity & $1(1)$ & $1(1)$ & 0.01065 \\
\hline Molecular function & Glucose-6-phosphate dehydrogenase activity & $1(1)$ & $1(1)$ & 0.01065 \\
\hline Molecular function & Deoxyribonuclease I activity & $1(1)$ & $1(1)$ & 0.01065 \\
\hline Molecular function & 3'-Phosphoadenosine $5^{\prime}$-phosphosulfate transmembrane transporter activity & $1(1)$ & $1(1)$ & 0.01065 \\
\hline Biological process & Olfactory behavior & $1(1)$ & $1(1)$ & 0.01091 \\
\hline Biological process & Positive regulation of dopamine receptor signaling pathway & $1(1)$ & $1(1)$ & 0.01091 \\
\hline Biological process & Regulation of dopamine receptor signaling pathway & $1(0)$ & $1(0)$ & 0.01091 \\
\hline Biological process & 3'-Phosphoadenosine 5'-phosphosulfate transport & $1(1)$ & $1(1)$ & 0.01091 \\
\hline Biological process & Negative regulation of neuron apoptosis & $2(2)$ & $28(28)$ & 0.01148 \\
\hline Cellular component & MHC protein complex & $2(0)$ & $26(0)$ & 0.01171 \\
\hline Molecular function & Toxin binding & $1(1)$ & $2(2)$ & 0.01594 \\
\hline Molecular function & $\mathrm{C} 2 \mathrm{H} 2$ zinc finger domain binding & $1(1)$ & $2(2)$ & 0.01594 \\
\hline Molecular function & Dopachrome isomerase activity & $1(1)$ & $2(2)$ & 0.01594 \\
\hline Molecular function & 6-Phosphogluconolactonase activity & $1(1)$ & $2(2)$ & 0.01594 \\
\hline Molecular function & Hedgehog receptor activity & $1(1)$ & $2(2)$ & 0.01594 \\
\hline Molecular function & Dihydrofolate reductase activity & $1(1)$ & $2(2)$ & 0.01594 \\
\hline Molecular function & Purine nucleoside transmembrane transporter activity & $1(0)$ & $2(1)$ & 0.01594 \\
\hline Biological process & Cytolysis by symbiont of host cells & $1(0)$ & $2(0)$ & 0.01632 \\
\hline Biological process & Cytolysis of cells in other organism during symbiotic interaction & $1(0)$ & $2(0)$ & 0.01632 \\
\hline Biological process & Cytolysis of cells of another organism & $1(0)$ & $2(0)$ & 0.01632 \\
\hline Biological process & Disruption by symbiont of host cells & $1(0)$ & $2(0)$ & 0.01632 \\
\hline Biological process & Hemolysis by symbiont of host erythrocytes & $1(1)$ & $2(2)$ & 0.01632 \\
\hline Biological process & Hemolysis of cells in other organism & $1(0)$ & $2(0)$ & 0.01632 \\
\hline Biological process & Hemolysis of cells in other organism during symbiotic interaction & $1(0)$ & $2(0)$ & 0.01632 \\
\hline Biological process & Killing by symbiont of host cells & $1(0)$ & $2(0)$ & 0.01632 \\
\hline Biological process & Maintenance of mitochondrion location & $1(1)$ & $2(2)$ & 0.01632 \\
\hline Biological process & $\begin{array}{c}\text { Modification by organism of cell membrane in other organism } \\
\text { during symbiotic interaction }\end{array}$ & $1(0)$ & $2(0)$ & 0.01632 \\
\hline Biological process & Modification by symbiont of host cell membrane & $1(0)$ & $2(0)$ & 0.01632 \\
\hline Biological process & Modification by symbiont of host cellular component & $1(0)$ & $2(0)$ & 0.01632 \\
\hline Biological process & Modification by symbiont of host structure & $1(0)$ & $2(0)$ & 0.01632 \\
\hline Biological process & $\begin{array}{l}\text { Modification of cellular component in other organism during } \\
\text { symbiotic interaction }\end{array}$ & $1(0)$ & $2(0)$ & 0.01632 \\
\hline Biological process & Modification of structure of other organism during symbiotic interaction & $1(0)$ & $2(0)$ & 0.01632 \\
\hline Biological process & Caveola assembly & $1(1)$ & $2(2)$ & 0.01632 \\
\hline Biological process & Membrane raft assembly & $1(0)$ & $2(0)$ & 0.01632 \\
\hline Biological process & Positive regulation of G-protein coupled receptor protein signaling pathway & $1(0)$ & $2(1)$ & 0.01632 \\
\hline Biological process & Chromatin-mediated maintenance of transcription & $1(1)$ & $2(2)$ & 0.01632 \\
\hline Biological process & Positive regulation of gene expression, epigenetic & $1(0)$ & $2(0)$ & 0.01632 \\
\hline Biological process & ncRNA catabolic process & $1(0)$ & $2(0)$ & 0.01632 \\
\hline Biological process & rRNA catabolic process & $1(1)$ & $2(2)$ & 0.01632 \\
\hline Biological process & Purine nucleoside transport & $1(0)$ & $2(1)$ & 0.01632 \\
\hline Biological process & Striated muscle cell differentiation & $2(1)$ & $38(5)$ & 0.0199 \\
\hline Biological process & Antigen processing and presentation & $2(2)$ & $38(32)$ & 0.0199 \\
\hline
\end{tabular}


TABle 4: Continued.

\begin{tabular}{|c|c|c|c|c|}
\hline Ontology & Term & Changed genes & Total genes & $P$ value \\
\hline Molecular function & Copper ion binding & $2(2)$ & $39(38)$ & 0.01996 \\
\hline Molecular function & Endodeoxyribonuclease activity, producing 5 -phosphomonoesters & $1(0)$ & $3(1)$ & 0.0212 \\
\hline Cellular component & Membrane & $24(17)$ & $2769(1768)$ & 0.02168 \\
\hline Biological process & Maintenance of organelle location & $1(0)$ & $3(0)$ & 0.02171 \\
\hline Biological process & Melanin biosynthetic process & $1(1)$ & $3(3)$ & 0.02171 \\
\hline Biological process & Melanin metabolic process & $1(0)$ & $3(0)$ & 0.02171 \\
\hline Biological process & Endoplasmic reticulum calcium ion homeostasis & $1(1)$ & $3(1)$ & 0.02171 \\
\hline Biological process & Multicellular organismal water homeostasis & $1(0)$ & $3(0)$ & 0.02171 \\
\hline Biological process & Renal water homeostasis & $1(1)$ & $3(1)$ & 0.02171 \\
\hline Biological process & Positive regulation of Rac protein signal transduction & $1(1)$ & $3(3)$ & 0.02171 \\
\hline Biological process & Apoptosis & $7(3)$ & $522(189)$ & 0.0221 \\
\hline Biological process & Programmed cell death & $7(0)$ & $525(2)$ & 0.02273 \\
\hline Cellular component & Extrinsic to internal side of plasma membrane & $1(1)$ & $3(3)$ & 0.02347 \\
\hline Cellular component & Spectrin & $1(1)$ & $3(3)$ & 0.02347 \\
\hline Molecular function & Endonuclease activity & $2(2)$ & $43(30)$ & 0.02378 \\
\hline Molecular function & Nucleoside transmembrane transporter activity & $1(0)$ & $4(1)$ & 0.02643 \\
\hline Biological process & Regulation of neuron apoptosis & $2(0)$ & $45(2)$ & 0.02693 \\
\hline Biological process & Detection of visible light & $1(1)$ & $4(1)$ & 0.02706 \\
\hline Biological process & Chemosensory behavior & $1(0)$ & $4(3)$ & 0.02706 \\
\hline Biological process & Protein maturation by protein folding & $1(1)$ & $4(4)$ & 0.02706 \\
\hline Biological process & Cellular chaperone-mediated protein complex assembly & $1(1)$ & $4(2)$ & 0.02706 \\
\hline Biological process & Mitochondrial outer membrane translocase complex assembly & $1(1)$ & $4(4)$ & 0.02706 \\
\hline Biological process & Outer mitochondrial membrane organization & $1(0)$ & $4(0)$ & 0.02706 \\
\hline Biological process & Glycine biosynthetic process & $1(1)$ & $4(2)$ & 0.02706 \\
\hline Cellular component & Membrane part & $21(0)$ & $2325(1)$ & 0.02771 \\
\hline Biological process & Cellular membrane organization & $4(0)$ & $212(30)$ & 0.02909 \\
\hline Biological process & Membrane organization & $4(0)$ & $212(0)$ & 0.02909 \\
\hline Biological process & Neuron apoptosis & $2(0)$ & $48(3)$ & 0.03021 \\
\hline Biological process & Neuron death & $2(0)$ & $48(0)$ & 0.03021 \\
\hline Biological process & Muscle cell differentiation & $2(0)$ & $49(1)$ & 0.03134 \\
\hline Biological process & Disruption of cells of other organism during symbiotic interaction & $1(0)$ & $5(0)$ & 0.03239 \\
\hline Biological process & Killing of cells in other organism during symbiotic interaction & $1(0)$ & $5(0)$ & 0.03239 \\
\hline Biological process & Water homeostasis & $1(0)$ & $5(1)$ & 0.03239 \\
\hline Biological process & Endoplasmic reticulum organization & $1(1)$ & $5(5)$ & 0.03239 \\
\hline Biological process & Membrane raft organization & $1(0)$ & $5(1)$ & 0.03239 \\
\hline Biological process & Pinocytosis & $1(1)$ & $5(2)$ & 0.03239 \\
\hline Biological process & Nucleoside transport & $1(0)$ & $5(2)$ & 0.03239 \\
\hline Biological process & Response to light stimulus & $2(0)$ & $51(8)$ & 0.03364 \\
\hline Biological process & Cell death & $7(0)$ & $578(68)$ & 0.03597 \\
\hline Biological process & Vesicle-mediated transport & $5(2)$ & $336(120)$ & 0.03622 \\
\hline Biological process & Death & $7(0)$ & $579(0)$ & 0.03626 \\
\hline Biological process & Positive regulation of signaling pathway & $3(0)$ & $133(0)$ & 0.03743 \\
\hline Biological process & Modification by host of symbiont morphology or physiology & $1(0)$ & $6(0)$ & 0.03769 \\
\hline Biological process & ER overload response & $1(1)$ & $6(5)$ & 0.03769 \\
\hline Biological process & Regulation of Rac protein signal transduction & $1(0)$ & $6(1)$ & 0.03769 \\
\hline Molecular function & Actin binding & $3(3)$ & $139(121)$ & 0.0394 \\
\hline Cellular component & HOPS complex & $1(1)$ & $6(6)$ & 0.04072 \\
\hline Molecular function & Intramolecular oxidoreductase activity, transposing $\mathrm{C}=\mathrm{C}$ bonds & $1(0)$ & $7(0)$ & 0.04196 \\
\hline Biological process & Detection of light stimulus & $1(0)$ & $7(0)$ & 0.04296 \\
\hline
\end{tabular}


TABLE 4: Continued.

\begin{tabular}{lcccc}
\hline Ontology & Term & Changed genes & Total genes & $P$ value \\
\hline Biological process & Metabotropic glutamate receptor signaling pathway & $1(1)$ & $7(4)$ & 0.04296 \\
Biological process & Regulation of synaptic transmission, GABAergic & $1(1)$ & $7(2)$ & 0.04296 \\
Cellular component & Internal side of plasma membrane & $1(0)$ & $7(4)$ & 0.04641 \\
Molecular function & Scavenger receptor activity & $1(1)$ & $8(8)$ & 0.04708 \\
Biological process & Interaction with symbiont & $1(0)$ & $8(1)$ & 0.0482 \\
Biological process & Modification by symbiont of host morphology or physiology & $1(0)$ & $8(0)$ & 0.0482 \\
Biological process & Chaperone-mediated protein complex assembly & $1(0)$ & $8(4)$ & 0.0482 \\
Biological process & Positive regulation of Ras protein signal transduction & $1(0)$ & $8(4)$ & 0.0482 \\
Biological process & Positive regulation of small GTPase-mediated signal transduction & $1(0)$ & $8(0)$ & 0.0482 \\
Biological process & Synaptic transmission, GABAergic & $1(0)$ & $8(1)$ & 0.0482 \\
Biological process & Actin filament capping & $1(1)$ & $8(6)$ & 0.0482 \\
Biological process & Pentose-phosphate shunt & $1(1)$ & $8(7)$ & 0.0482 \\
Biological process & Calcium ion transport & $2(1)$ & $63(41)$ & 0.04876 \\
Cellular component & Intrinsic to membrane & $17(0)$ & $1867(20)$ & 0.0491 \\
\hline
\end{tabular}

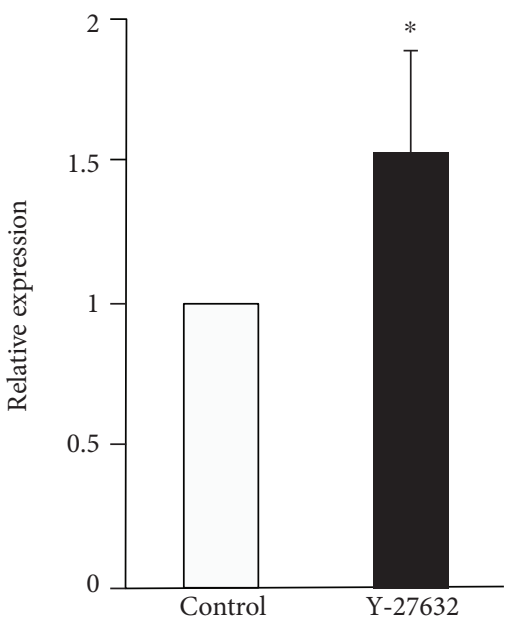

(a)

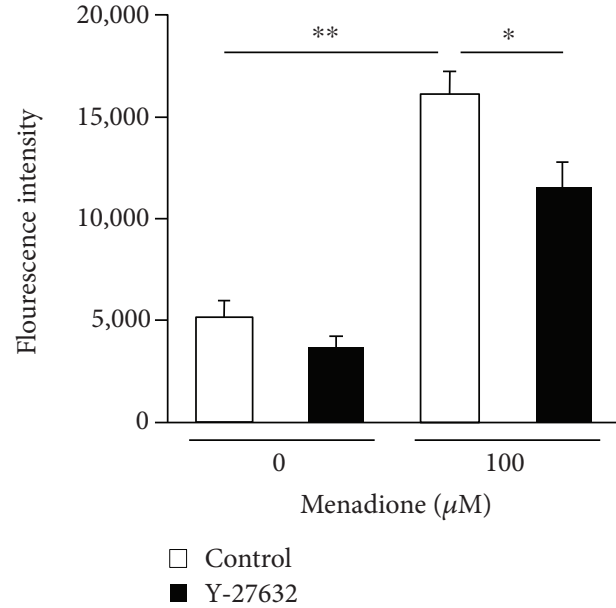

(b)

FIgure 1: (a) Quantitative PCR analysis of catalase mRNA. The TM cells were treated with $25 \mu \mathrm{M}$ Y-27632 for 30 min. The relative expression

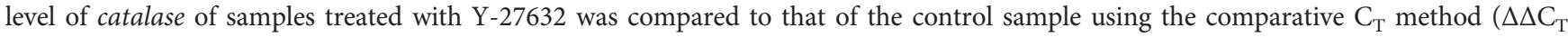
method). The $18 \mathrm{~S}$ ribosomal RNA was used as an endogenous control. Data are shown as mean \pm SE from six independent experiments. ${ }^{*} P<0.05$ compared with control by Wilcoxon rank sum test. (b) The effects of Y-27632 on the intracellular production of reactive oxygen species (ROS). The TM cells were treated with or without $25 \mu \mathrm{M} \mathrm{Y}-27632$ for $30 \mathrm{~min}$, followed by $100 \mu \mathrm{M}$ menadione stimulated for $1 \mathrm{~h}$. ROS were detected by CellROX reagent, and the fluorescence of the TM cells were measured by cell sorter SH800. Data are shown as mean \pm SE from five independent experiments. ${ }^{* *} P<0.01$ and ${ }^{*} P<0.05$ compared with control by the Wilcoxon rank sum test (a) and Tukey Kramer HSD test (b).

reported that Y-27632 induced p-53-mediated apoptosis in hemangioma [26]. In the present study, we indicated that ROCK inhibitor effected cell survival in TM cells. This is interesting point since ROCK inhibitor-induced effects such as cell death or cell protection were changed by differences of cell types.

TM has a critical role in the maintenance of aqueous outflow resistance through the regulation of extracellular matrix metabolism, phagocytosis of debris, and empty space associated with tissue contraction [27, 28]. Indeed, the number of TM cells is decreased in glaucomatous eyes [29], suggesting that functional TM cells are essential in controlling IOP. In this context, oxidative stress is a potential cause of cellular dysregulation in TM, both functionally and numerally, because it has been suggested that the TM of glaucomatous eyes is continuously exposed to oxidative 


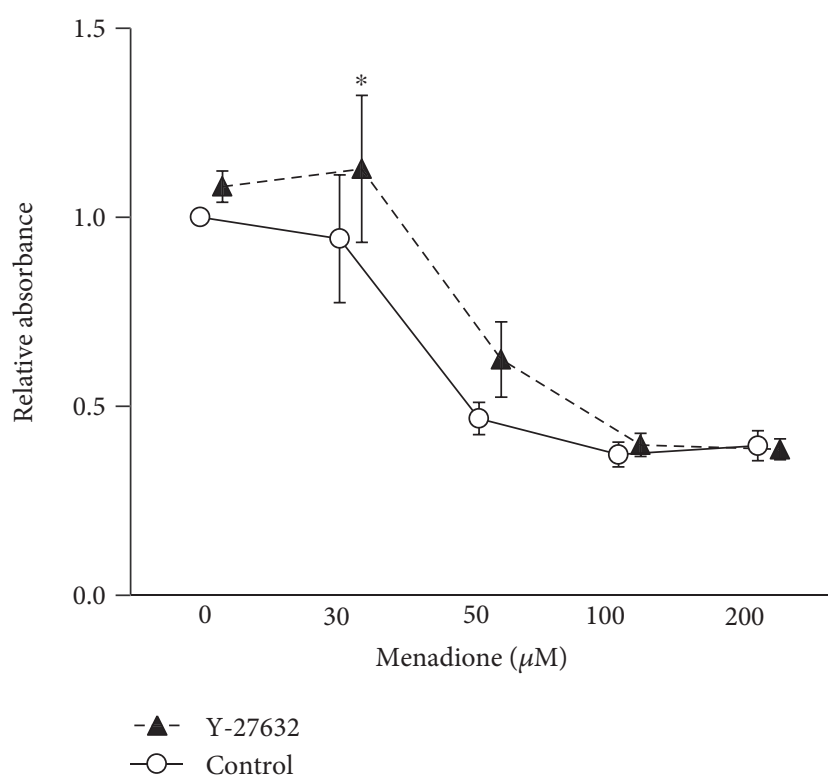

(a)

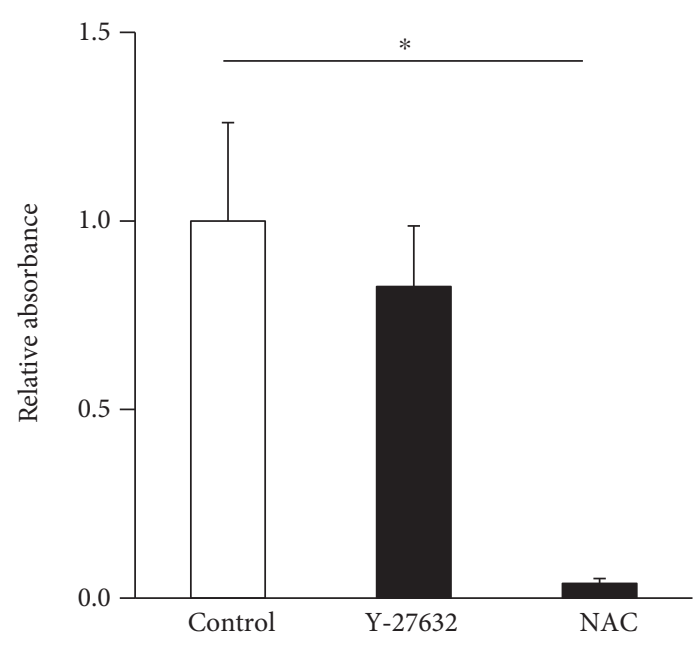

(b)

FIgURE 2: (a) The effect of Y-27632 on oxidative stress-induced cell death. The TM cells were treated with or without $25 \mu \mathrm{M}$ Y-27632 for $30 \mathrm{~min}$, followed by menadione stimulation of the cells for $24 \mathrm{~h}$. Cell viabilities were shown as relative value compared with the control. Data are shown as the mean \pm SE from six independent experiments. ${ }^{*} P<0.05$ compared with control by Wilcoxon rank sum test. (b) The effect of Y-27632 on extracellular antioxidative activity. The xanthine-oxidase-induced superoxide production was assessed using a superoxide-sensitive luminescent reagent. Data are shown as the mean \pm SE from six independent experiments. ${ }^{*} P<0.05$ compared with the control by Dunnett's test. Nac: n-acetyl cysteine.

stress [2-7]. Thus, an antioxidant drug might reduce oxidative stress in TM cells, slowing progression of glaucomatous damage in outflow tissues. Though it remains unknown whether clinically used eye-drops containing ripasudil have significant antioxidative effects on TM cells in vivo, the present study's findings may be clinically relevant.

The effect of Y-27632 on cell survival under oxidative stress was significant, but limited. Since glaucoma progresses chronically in the majority of the patients, the acute oxidative damage in the present study may not reflect pathological conditions in glaucomatous TM cells, which is one of the limitations of the present study. Another limitation is that the antioxidative effects of ROCK inhibition were not corroborated in vivo. Further studies are required to acquire more clinically relevant evidence of the effects of ROCK inhibitor on oxidative stress in TM.

\section{Conclusion}

Microarray analysis reveals that Y-27632 upregulates antioxidative genes including catalase and partially reduces the ROS production and cell death by oxidative stress induced by menadione.

\section{Conflicts of Interest}

Dr. Hidenobu Tanihara has received consulting fees from Kowa and MSD and board membership fees from Senju Pharmaceutical, Santen Pharmaceutical, Alcon Japan, and Pfizer Japan.

\section{Acknowledgments}

This work was supported by the JSPS KAKENHI Grant nos. $26293375,15 \mathrm{~K} 15636$, and 26462664.

\section{References}

[1] M. A. Babizhayev, K. S. Vishnyakova, and Y. E. Yegorov, "Oxidative damage impact on aging and age-related diseases: drug targeting of telomere attrition and dynamic telomerase activity flirting with imidazole-containing dipeptides," Recent Patents on Drug Delivery \& Formulation, vol. 8, no. 3, pp. 163-192, 2014.

[2] S. M. Ferreira, S. F. Lerner, R. Brunzini, P. A. Evelson, and S. F. Llesuy, "Oxidative stress markers in aqueous humor of glaucoma patients," American Journal of Ophthalmology, vol. 137, no. 1, pp. 62-69, 2004.

[3] A. A. Ghanem, L. F. Arafa, and A. El-Baz, "Oxidative stress markers in patients with primary open-angle glaucoma," Current Eye Research, vol. 35, no. 4, pp. 295-301, 2010.

[4] V. Zanon-Moreno, P. Marco-Ventura, A. Lleo-Perez et al., "Oxidative stress in primary open-angle glaucoma," Journal of Glaucoma, vol. 17, no. 4, pp. 263-268, 2008.

[5] A. Goyal, A. Srivastava, R. Sihota, and J. Kaur, "Evaluation of oxidative stress markers in aqueous humor of primary open angle glaucoma and primary angle closure glaucoma patients," Current eye Research, vol. 39, no. 8, pp. 823-829, 2014.

[6] A. Izzotti, S. C. Sacca, C. Cartiglia, and S. De Flora, "Oxidative deoxyribonucleic acid damage in the eyes of glaucoma patients," American Journal of Medicine, vol. 114, no. 8, pp. 638-646, 2003. 
[7] S. C. Sacca, A. Pascotto, P. Camicione, P. Capris, and A. Izzotti, "Oxidative DNA damage in the human trabecular meshwork: clinical correlation in patients with primary open-angle glaucoma," Archives of Ophthalmology, vol. 123, no. 4, pp. 458-463, 2005.

[8] M. Tanito, S. Kaidzu, Y. Takai, and A. Ohira, "Correlation between systemic oxidative stress and intraocular pressure level," PLoS One, vol. 10, no. 7, article e0133582, 2015.

[9] Y. Tang, E. A. Scheef, S. Wang et al., "CYP1B1 expression promotes the proangiogenic phenotype of endothelium through decreased intracellular oxidative stress and thrombospondin2 expression," Blood, vol. 113, no. 3, pp. 744-754, 2009.

[10] Y. Zhao, S. Wang, C. M. Sorenson et al., "Cyp1b1 mediates periostin regulation of trabecular meshwork development by suppression of oxidative stress," Molecular and Cellular Biology, vol. 33, no. 21, pp. 4225-4240, 2013.

[11] Y. A. Ito, I. S. Goping, F. Berry, and M. A. Walter, "Dysfunction of the stress-responsive FOXC1 transcription factor contributes to the earlier-onset glaucoma observed in Axenfeld-Rieger syndrome patients," Cell Death \& Disease, vol. 5, article e1069, 2014.

[12] F. B. Berry, J. M. Skarie, F. Mirzayans et al., "FOXC1 is required for cell viability and resistance to oxidative stress in the eye through the transcriptional regulation of FOXO1A," Human Molecular Genetics, vol. 17, no. 4, pp. 490-505, 2008.

[13] M. Honjo, H. Tanihara, M. Inatani et al., "Effects of rhoassociated protein kinase inhibitor Y-27632 on intraocular pressure and outflow facility," Investigative Ophthalmology and Visual Sciences, vol. 42, no. 1, pp. 137-144, 2001.

[14] P. V. Rao, P. F. Deng, J. Kumar, and D. L. Epstein, "Modulation of aqueous humor outflow facility by the rho kinasespecific inhibitor Y-27632," Investigative Ophthalmology and Visual Sciences, vol. 42, no. 5, pp. 1029-1037, 2001.

[15] K. P. Garnock-Jones, "Ripasudil: first global approval," Drugs, vol. 74, no. 18, pp. 2211-2215, 2014.

[16] H. Tanihara, T. Inoue, T. Yamamoto et al., "Phase 2 randomized clinical study of a rho kinase inhibitor, K-115, in primary open-angle glaucoma and ocular hypertension," American Journal of Ophthalmology, vol. 156, no. 4, pp. 731-736, 2013.

[17] H. Tanihara, T. Inoue, T. Yamamoto et al., "Additive intraocular pressure-lowering effects of the rho kinase inhibitor ripasudil (K-115) combined with timolol or latanoprost: a report of 2 randomized clinical trials," JAMA Ophthalmology, vol. 133, no. 7, pp. 755-761, 2015.

[18] T. Nishikimi and H. Matsuoka, "Molecular mechanisms and therapeutic strategies of chronic renal injury: renoprotective effect of rho-kinase inhibitor in hypertensive glomerulosclerosis," Journal of Pharmacological Sciences, vol. 100, no. 1, pp. 22-28, 2006.

[19] K. Satoh, Y. Fukumoto, and H. Shimokawa, "Rho-kinase: important new therapeutic target in cardiovascular diseases," American Journal of Physiology Heart and Circulatory Physiology, vol. 301, no. 2, pp. H287-H296, 2011.

[20] K. Yamamoto, K. Maruyama, N. Himori et al., "The novel rho kinase (ROCK) inhibitor K-115: a new candidate drug for neuroprotective treatment in glaucoma," Investigative Ophthalmology and Visual Sciencies, vol. 55, no. 11, pp. 7126-7136, 2014.

[21] T. Fujimoto, T. Inoue, T. Kameda et al., "Involvement of RhoA/Rho-associated kinase signal transduction pathway in dexamethasone-induced alterations in aqueous outflow,"
Investigative Ophthalmology and Visual Sciences, vol. 53, no. 11, pp. 7097-7108, 2012.

[22] M. Inoue-Mochita, T. Inoue, T. Fujimoto et al., “p38 MAP kinase inhibitor suppresses transforming growth factor-beta2induced type 1 collagen production in trabecular meshwork cells," PLoS One, vol. 10, no. 3, article e0120774, 2015.

[23] T. Kameda, T. Inoue, M. Inatani et al., "The effect of rhoassociated protein kinase inhibitor on monkey Schlemm's canal endothelial cells," Investigative Ophthalmology and Visual Sciences, vol. 53, no. 6, pp. 3092-3103, 2012.

[24] N. Awai-Kasaoka, T. Inoue, T. Kameda, T. Fujimoto, M. Inoue-Mochita, and $\mathrm{H}$. Tanihara, "Oxidative stress response signaling pathways in trabecular meshwork cells and their effects on cell viability," Molecular Vision, vol. 19, pp. 13321340, 2013.

[25] K. Satoh, P. Nigro, T. Matoba et al., "Cyclophilin A enhances vascular oxidative stress and the development of angiotensin II-induced aortic aneurysms," Nature Medicine, vol. 15, no. 6, pp. 649-656, 2009.

[26] M. K. Qiu, S. Q. Wang, C. Pan et al., "ROCK inhibition as a potential therapeutic target involved in apoptosis in hemangioma," Oncology Reports, vol. 37, no. 5, pp. 2987-2993, 2017.

[27] U. Roy Chowdhury, C. R. Hann, W. D. Stamer, and M. P. Fautsch, "Aqueous humor outflow: dynamics and disease," Investigative Ophthalmology and Visual Sciences, vol. 56, no. 5, pp. 2993-3003, 2015.

[28] J. A. Vranka, M. J. Kelley, T. S. Acott, and K. E. Keller, "Extracellular matrix in the trabecular meshwork: intraocular pressure regulation and dysregulation in glaucoma," Experimental eye Research, vol. 133, pp. 112-125, 2015.

[29] J. Alvarado, C. Murphy, and R. Juster, “Trabecular meshwork cellularity in primary open-angle glaucoma and nonglaucomatous normals," Ophthalmology, vol. 91, no. 6, pp. 564579, 1984. 


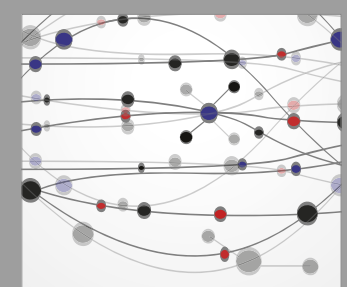

The Scientific World Journal
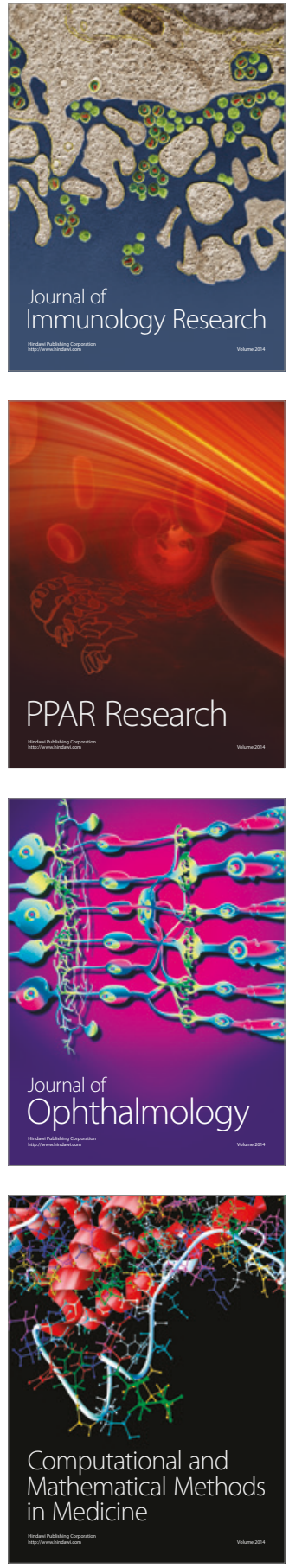

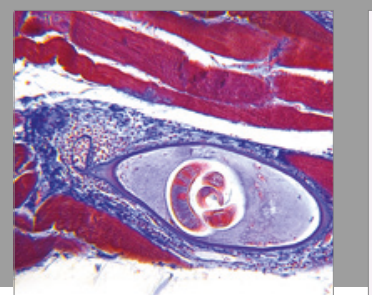

Gastroenterology Research and Practice
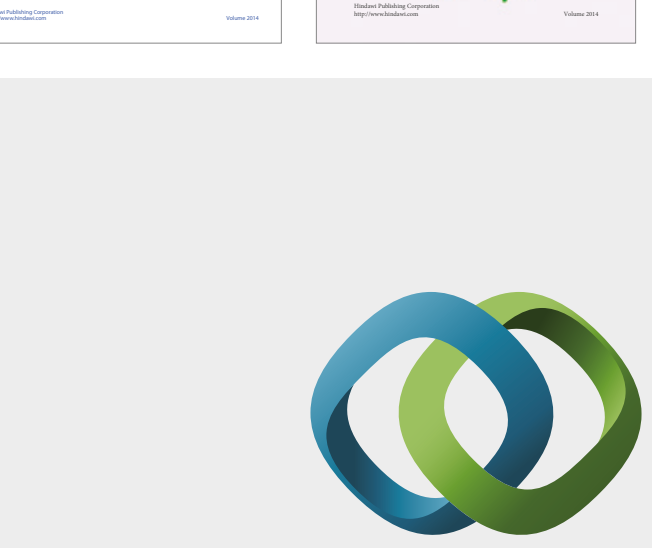

\section{Hindawi}

Submit your manuscripts at

https://www.hindawi.com
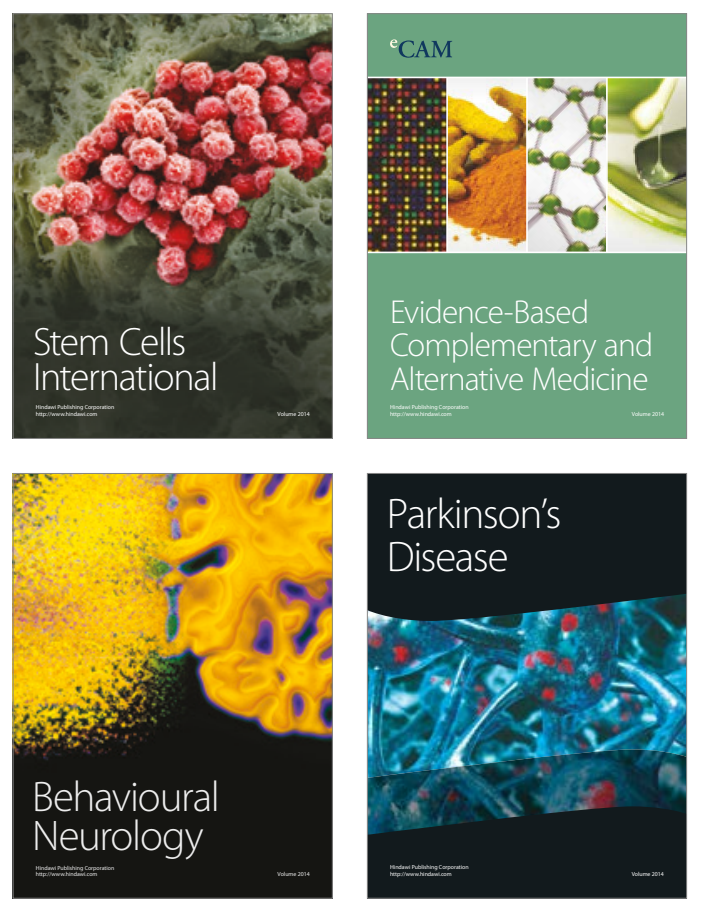
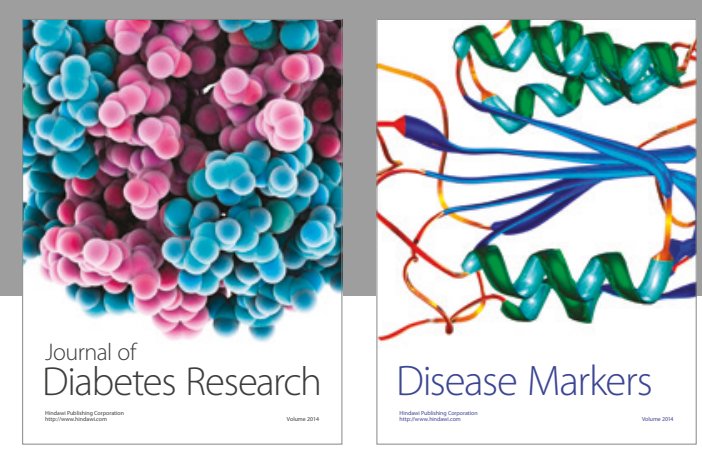

Disease Markers
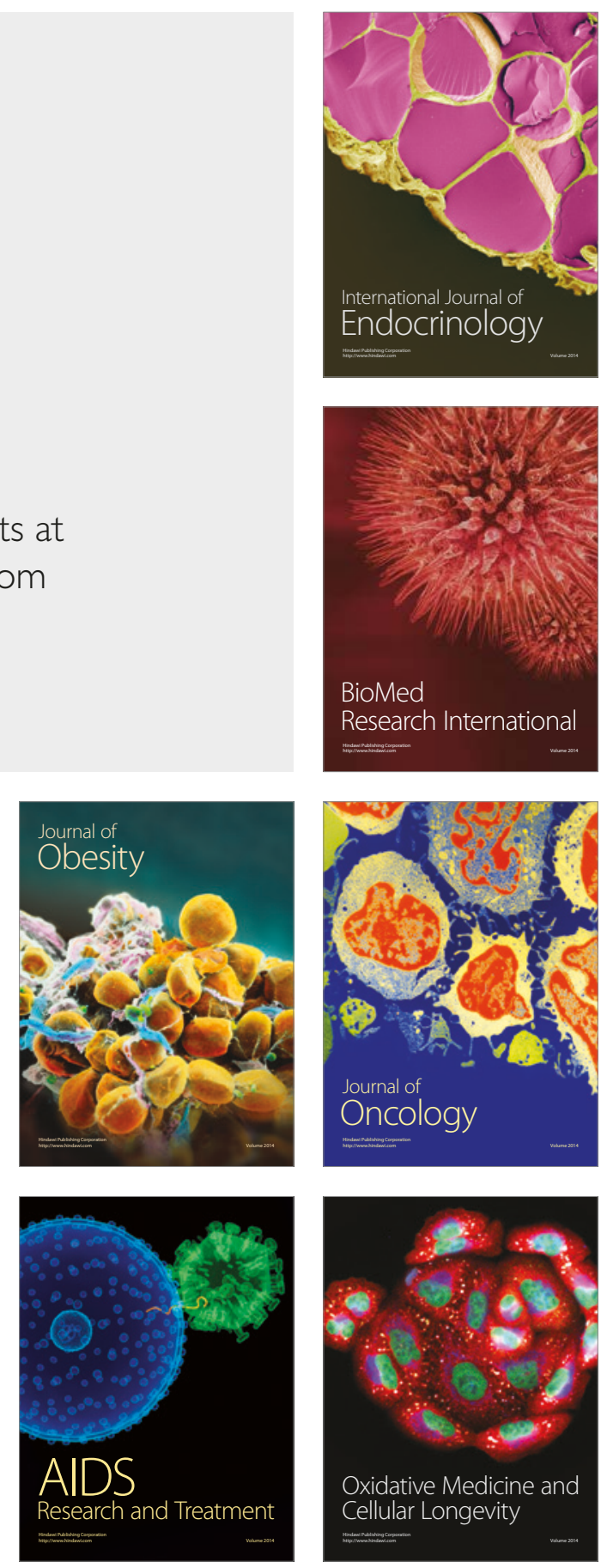